\title{
Die standörtliche Verbreitung und klimaökologische Abgrenzung waldbildender submediterraner (Quercus pubescens), subborealer (Pinus silvestris) und mitteleuropäisch-montaner Florenelemente (Abies alba) am Beispiel des mittleren Wallis (Zentralalpen)
}

\section{Einleitung und Problemstellung}

Es muss als eine bedeutsame Frage sowohl der Vegetations - als auch der Klimageographie angesehen werden, ob Klimafaktoren bzw. bestimmte Elemente des Klimas zur Erklärung des Verbreitungsmusters von bestimmten Arten benutzt werden können. Für die Beziehungen $z w i s c h e n$ Klima, insbesondere den Niederschlagsverhältnissen, und Vegetationsformationen sind vor allem durch die Arbeiten von TROLL $(1952 ; 1957 ; 1961)$ und LAUER (1952) grundsätzliche und weitgehend abgesicherte Ergebnisse gewonnen worden, da hierbei der physiognomische Gesamteindruck der Vegetation - über alle einzelnen bestandsbildenden Arten hinweg - das entscheidende Kriterium darstellt.

Bei der Beurteilung des Verbreitungsmusters von Arten in ihrer Beziehung zum Klima bestehen dagegen eine Reihe von grundsätzlichen Schwierigkeiten, die in den folgenden Punkten zusammengefasst werden können:

1) Die unterschiedliche Florenentwicklung in den verschiedenen Erdräumen ist in erster Linie historisch bedingt und hängt mit einer weit in die Vergangenheit reichenden Isolierung dieser Grossräume zusammen. Es kommt hinzu, dass diese historische Entwicklung auch von paläoklimatischen Veränderungen, z.B. den Eiszeiten, in unterschiedlichem Umfange beeinträchtigt wurde und daher auch nicht immer von rezenten klimaökologischen Gegebenheiten zu klären ist.

2) Obwohl es viele Arten gibt, die ein bestimmtes Klima zu repräsentieren scheinen, so sind dennoch in der Mehrzahl der Fälle Arealverbreitungskarten und Klimakarten, meist auch nur kartographische Darstellungen eines bestimmten Klimafaktors, nicht identisch.

3) Die entscheidenden Konkur renzverhältnisse zwischen den Arten können auf Grund der unterschiedlichen florengeschichtlichen und arealkundlichen Zusammhänge sowie des grossen Spektrums gesellschaftlicher Kombinationsmöglichkeiten auch in konvergenten Lebensräumen unterschiedlich sein.

Der bisherige Erkenntnisstand der in dieser Arbeit gesetzten Fragestellung scheint immer noch in den von KOEPPEN (1931) erarbeiteten Prinzipien der effektiven Klassifikation der Klimate zu bestehen, wonach Pflanzenarten, insbesondere bestandsbildende Baumarten, die beobachteten Klimawirkungen darstellen (z. B. Buchenklima Cfb). Die entsprechenden Klimaelemente sind klar definiert durch Schwellen - und Andauerwerte, die mess- oder einwandfrei beobachtbar sind und in ausreichender Dichte vorliegen. $\mathrm{Zu}$ diesen Ergebnissen ist folgendes zu bemerken:

Die Klimaklassifikation von KOEPPEN unter Berücksichtigung von Arten ist ein Ergebnis der klassischen Mittelwertsklimatologie. Es muss nun aber auch zumindest der Versuch lohnenswert erscheinen, ob etwa Häufigkeitsanalysen monatlicher Niederschlagssummen in eine öko-physiologische und räumlich fassbare Beziehung zum Verbreitungsmuster von Arten gebracht werden können. Dies muss um so mehr gelten, da hierdurch - und nicht etwa durch die monatlichen Mittelwerte - die Wahrscheinlichkeit ausgedrückt werden kann, mit der und in welchem Umfang es in einem für das pflanzliche Wachstum wichtigen Zeitabschnitt, insbesondere der sommerlichen Vegetationsperiode, regnet. Auch von der Mittelwertsklimatologie abweichende Frequenzanalysen von Trockenperioden können etwa für die Verbreitung von mesophilen oder xerischen Arten Aufschlüsse geben und damit eine hohe vegetationsökologische Wertigkeit besitzen. Die Einstrahlungssummen, welche bisher in vegetationsgeographischen Arbeiten kaum berücksichtigt wurden, können ebenfalls in räumlich - differenzierender Weise zur Erklärung des Verbreitungsmusters von bestimmten Arten - in dieser Arbeit der Flaumeiche - benutzt werden. Freilich wird sich auch in dieser Arbeit erweisen, dass zusätzlich zu den oben genannten Punkten auf die Verwendung von gewissen Elementen der Mittelwertsklimatologie nicht verzichtet werden kann, da weiterführende Beobachtungen ausserhalb des zur Verfügung stehenden Stationsnetzes nicht vorliegen. Es wird sich auch gerade als vegetationsgeographisch vorteilhaft erweisen, durch den räum-

Prof.Dr.Norbert Stein, Geographisches Institut Universität Saarland, Abteilung Biogeographie Postfach, D - 66 Saarbrücken 
lichen Vergleich mit anderen europäischen Vorkommensbereichen der hier behandelten Baumarten die im Wallis gewonnenen Erkenntnisse abzusichern und zu erweitern. Ein wesentliches Ziel dieser Arbeit besteht darin aufzuzeigen, ob es mit Hilfe des von Stationen gelieferten Beobachtungsmaterials mög lich ist, entscheidend über die methodischen Ergebnisse von KOEPPEN hinauszugelangen oder ob wesentlich verfeinerte Methoden nicht mehr abhängig von den eingerichteten Stationen - für eine detaillierte ökologische Analyse erforderlich sind.

Wenn man daher die Verbreitung von Florenelementen - neben der so überaus wichtigen Bedeutung der historischen Arealgestaltung von den jeweils gegebenen klimaökologischen Voraussetzungen her erklären will, so erscheinen von der pflanzengeographischen Komponente her folgende Bedingungen erfüllt sein zu müssen:

1) Der mögliche Untersuchungsraum sollte eine einheitliche und, überschaubare Florenentwicklung besitzen, um deren Einfluss auf die räumliche Verteilung der Arten eindeutig bewerten zu können.

2) Es sollten in diesem Raum verschiedene Florenelemente vorhanden sein, deren arealmässiger Schwerpunkt nach den Verbreitungskarten in unterschiedlichen klimatischen Bereichen sich befindet.

3) Die in Frage kommenden Arten sollten jeweils für sich - im Grenzbereich ihres Gesamtareals stehen, da eben hier potentielle $\mathrm{Ge}$ setzmässigkeiten der ökologischen Valenz in der Regel schärfer zu fassen sind als im Zentrum des Areals. Dies gilt umso mehr, wenn bestimmte Arten entlang von - im einzelnen stets zu definierenden - räumlich-ökologischen Gradienten durch andere ersetzt werden, etwa sommergrüne Laubhölzer durch immergrüne oder auch mesophile Schattholzarten durch xerophile Lichtarten.

Ein Raum, der diesen Anforderungen entspricht, ist das mittlere Wallis in den Zentralalpen, wo als bestandsbildende Baumarten die submediterrane Flaumeiche (Quercus pubescens), die subboreale Föhre (Pinus silvestris) und die mitteleuropäisch-montane Weisstanne (Abies alba) vorkommen. Die in Fig. 3 enthaltenen weiteren Baumarten (Rotbuche; Fichte; Lärche; Arve; Bergkiefer) sind für die Darlegung der vollständigen Bestandszusammensetzung erfasst worden, werden in diesem Beitrag sonst aber nicht weiter berücksichtigt.

II. Grossräumige Arealverbreitung von Flaumeiche, Föhre und Weisstanne

Die Flaumeiche (Quercus pubescens) gilt vegetationsökologisch als typischer Vertreter des submediter ranen Florengebietes, was durch die Arealverbreitungskarte bestätigt wird (Fig. 1).
Sie löst die an extreme sommerliche Trockenheit und damit länger anhaltenden jahreszeitlichen Wasserstress sowie gleichzeitig an milde, frostfreie Winter ohne eine physiologische Notwendigkeit des Laubabwurfs angepasste immergrüne Vegetation von Eichengesellschaften mit starken xeromorphen Merkmalen ab, unter denen die Quercetalia ilicis mit der Steineiche (Quercus ilex)als Cha rakterbaum an prominenter Stelle stehen. Diese eigentliche eumediterrane Florenregion nimmt nur die unterste Stufe im Vertikalprofil der Mittelmeerländer ein (MARKGRAF 1942; RIKLI 1946; HORVAT 1962).

Schon in der submontanen Stufe vieler mediterraner Gebirge sowie nördlich an das Hartlaubgebiet angrenzend tritt ein auffallender Wechsel ein, indem die immergrünen Hartlaubhölzer verschwinden und durch sommergrüne abgelöst werden, unter denen Flaumeichenwälder (Quercetalia pubescentis) mit der Flaumeiche (Quercus pubescens) als Charakterart vorherrschen 1).

Die Föhre (auch Waldföhre oder Waldkiefer; Pinus silvestris) gehört zum euro-sibirischen Teil des borealen Florengebietes. Obwohl hier zweifellos der arealmässige Schwerpunkt vorhanden ist (Fig. 1), greift sie weit über den borealen Gürtel hinaus und kann selbst noch, wenn auch nur streng lokal, an der Dnjeprmündung am Ufer des Schwarzen Meeres, auf der Krim im Jaila-Gebirge (ssp. hamata; KUEMMEL 1949) und in der Sierra Nevada in S-Spanien (ssp. nevadensis) angetroffen werden, so dass von WALTER/STRAKA (1970) für P. silvestris die Bezeichnung "subboreales Florenelement" vorgeschlagen wurde.

Das Areal der von der Weisstanne (Abies al ha) beherrschten Gesellschaften ist nahezu ganz auf Mitteleuropa und einige klimatisch entsprechende Gebirge des submediterranen Europa beschränkt, die auch sonst durch mitteleuropäische Florenelemente ausgezeichnet sind (MATTFELD 1926). Das Areal spiegelt im allgemeinen den Verlauf der europäischen Gebirge wider. Die Weisstanne wird in der Regel als Mischholz angetroffen. Dabei ist es sowohl von pflanzensoziologischer als auch von vegetationsgeographischer Bedeutung, dass die Weisstanne in ihren ökologischen Standortsbeziehungen sehr viele Gemeinsamkeiten mit der Rotbuche (Fagus silvatica) besitzt. Mischbestände von Abies alba und F. silvatica sind häufig, und die Weisstanne gehört meist schon physiognomisch zu einer Vielzahl von Buchenwäldern, was auch durch tabellarische Vegetationsaufnahmen von der pflanzensoziologischen Seite her unterstützt wird. Es ist daher auch nur allzu berechtigt, dass die meisten Autoren Abies alba als einen Charakterbaum des Fagion Verbandes oder der Ordnung Fagetalia ansehen (in Zusammenfassung ELLENBERG 1963). 
III. Die räumlich - regionale und klimaökologische Analyse von Flaumeiche, Föhre und Weisstanne im mittleren Wallis

Das allseitig von mächtigen Gebirgsketten umschlossene inneralpine Rhonetal (Fig. 2) ist eine klimatische und biotische Einheit, welche es recht erheblich von den anderen Teilen der Schweizer Alpen unterscheidet. RIKLI (1946) bespricht in seinem klassischen Werk über die Vegetation des Mittelmeergebietes auch das Wallis. Auch in vielen anderen Darstellungen wird stets auf den "südlichen" Florencharakter hingewiesen (CHRIST 1919-20; BRAUN-BLANQUET 1961).

Die für die Ausprägung des Pflanzenkleides und für die klimaökologisch-räumliche Gliederung der Vegetation entscheidende Voraussetzung besteht darin, dass die hohen Ketten der nördlichen Berner und südlichen Walliser Alpen das mittlere Wallis von den grossräumigen Klima - und Wettereinflüssen isolieren und auf diese Weise die Ausbildung eines besonderen Talklimas ermöglichen, das - soweit das Haupttal betroffen ist - in seinen wesentlichen Zügen durch relative Niederschlagsarmut, hohe Sonneneinstrahlung (Tab. 1) und geringe Bewölkung charakterisiert ist.

1. Die Flaumeichenbestände - standörtliche Verbreitung und deren klimaökologische Ursachen

Die Flaumeichenbestände haben sehr starke räumliche Reduzierungen erfahren, da ihre natürliche Verbreitungsstufe unterhalb von $1300 \mathrm{~m}$ zum Wirtschaftsraum des dicht besiedelten Walliser Haupttales gehört. Dennoch lassen sich die Reliktvorkommen jener früher ausgedehnteren Flaumeichenwäl. der ganz bestimmten Standorttypen, welche häufig auch als physiographische und pflanzensoziologisch abgrenzbare Oekotopen definiert werden können, zuordnen.

Bei der quantitativen Erfassung der Flaumeichen, welche mit Hilfe von Profillinien in Fig. 3 zur Darstellung gebracht wird, konnte im $\mathrm{Ge}$ lände die Verfolgung einer "Linie" wegen der starken anthropogenen Einflüsse nicht eingehalten werden, sondern es mussten auf breiten, häufig bis zu $3 \mathrm{~km}$ breiten "Bändern" die verbliebenen, oft kümmerlichen Waldbestände zusammengefasst werden. Dies gilt auch für die anderen Baumarten. In diesem Sinne muss auch die in Fig. 2 eingetragene Lage der Profillinien verstanden werden. Das in Fig. 3 dargestellte Verhältnis der Baumarten zueinander berücksichtigt nicht etwa vorhandene weitere Baumarten wie insbesondere Birke (Betula pendula), Grünerle (Alnus viridis) u. a., deren Anteil an der Gesamtzusammensetzung der Bestände in der Regel jedoch weniger als $5 \%$ beträgt.

1. 1. Abhängigkeit von Exposition und Einstrahlungssummen

\begin{tabular}{|c|c|c|c|c|c|c|c|c|c|c|c|c|c|c|c|c|c|c|}
\hline Sion & & $\mathrm{J}$ & F & $M$ & A & M & $\mathrm{J}$ & $\mathrm{J}$ & A & $S$ & 0 & $\mathrm{~N}$ & D & 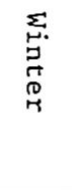 & 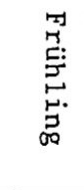 & $\begin{array}{l}n \\
0 \\
03 \\
⿱ 50 \\
0 \\
n\end{array}$ & 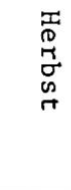 & 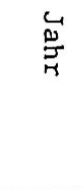 \\
\hline \multirow{3}{*}{$\begin{array}{l}(549 \mathrm{~m} \\
=7^{\circ} 22^{\prime} \\
\left.=46^{\circ} 14^{\prime}\right) \\
1940-1960 \mathrm{c}\end{array}$} & & 102 & 123 & 175 & 199 & 208 & 228 & 262 & 234 & 191 & 162 & 112 & 98 & 323 & 582 & 724 & 465 & 2094 \\
\hline & & 239 & 246 & 312 & 357 & 411 & 422 & 416 & 390 & 322 & 284 & 241 & 225 & 710 & 1080 & 1228 & 847 & 3865 \\
\hline & & 43 & 50 & 56 & 56 & 51 & 54 & 63 & 60 & 59 & 57 & 46 & 44 & 45 & 54 & 59 & 55 & 54 \\
\hline \multicolumn{19}{|l|}{ Montana } \\
\hline \multirow{3}{*}{$\begin{array}{rl} & (1453 \mathrm{~m} \mathrm{a} \\
= & 7^{\circ} 29^{\prime} \\
= & \left.46^{\circ} 19^{\prime}\right) \\
1 & b 26-1960 \mathrm{c}\end{array}$} & & 119 & 131 & 180 & 193 & 206 & 224 & 256 & 236 & 296 & 173 & 124 & 115 & 365 & 579 & 716 & 493 & 2153 \\
\hline & & 256 & 268 & 338 & 368 & 415 & 419 & 425 & 398 & 345 & 315 & 264 & 246 & 770 & 1121 & 1242 & 924 & 4057 \\
\hline & & 46 & 49 & 53 & 52 & 50 & 53 & 60 & 59 & 57 & 55 & 47 & 47 & 47 & 52 & 58 & 53 & 53 \\
\hline \multicolumn{19}{|l|}{ Zürich } \\
\hline \multirow{3}{*}{ 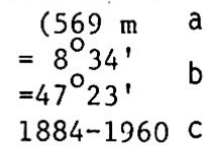 } & & 46 & 79 & 149 & 173 & 207 & 220 & 238 & 219 & 166 & 108 & 51 & 37 & 162 & 529 & 677 & 325 & 1693 \\
\hline & & 249 & 265 & 345 & 385 & 435 & 444 & 447 & 416 & 355 & 314 & 253 & 237 & 751 & 1165 & 1307 & 922 & 4145 \\
\hline & & 18 & 30 & 43 & 45 & 48 & 50 & 53 & 5.3 & 47 & 34 & 20 & 16 & 22 & 45 & 52 & 35 & 41 \\
\hline
\end{tabular}

Tab. 1:

a) mittlere Sonnenscheindauer

b) mögliche Sonnenscheindauer c) relative Sonnenscheindauer (in \% der möglichen Sonnenscheindauer)

Mittelwerte der Sonnenscheindauer im mittleren Wallis; zum Vergleich Zürich im nördlichen Alpenvorland (nach SCHUEPP 1962) 
Das räumliche Anordnungsmuster be züglich der Vorkommenshäufigkeit (Fig. 3 und 4) zeigt eine deutliche Konzentration der Flaumeichen auf die Sexponierten Hänge der nördlichen Flanken des Haupttales, wo ihre Frequenz bis zu $45 \%$ am $\mathrm{Ge}-$ samtbestand betragen kann. Es ist weiterhin wichtig, dass die obere Grenze der Vorkommen auf der S-Seite des Haupttales (=N-Exposition) bei 800-900 $\mathrm{m}$ deutlich tiefer liegt als auf der $\mathrm{N}$-Seite, wo Q. pubescens in grösserer Häufigkeit bis zu $1300 \mathrm{~m}$ vorkommt und Einzelindividuen sogar bis zu $1600 \mathrm{~m}$ beobachtet werden konnten. Die entscheidende Bedeutung der Expositionslage und damit der Dauer der täglichen Sonneneinstrahlung kann vor allem in Bereichen mit stark gegliedertem Relief und kleinräumig schnell wechselnder Expositionen erkannt werden, wie es häufig am Ausgang der Seitentäler in das Haupttal der Fall ist. So nehmen von Flaumeichen dominierte Bestände auf der östlichen Seite des unteren Vispertales (Fig. 5) die nach SW exponierten Lagen bis etwa $1000 \mathrm{~m}$ ein, während in N-und NW-Expositionen sowie in Lagen zwischen $1000 \mathrm{~m}$ und $1400 \mathrm{~m}$ Föhrenbestände mit relativ wenigen Vorkommen von Flaumeichen vorkommen. Die Kammlinie bildet dabei in der Regel eine recht markante Trennungslinie zwischen den beiden Waldgesellschaften. Es muss als eine ökologische Konvergenz interpretiert werden, dass der Weinanbau hier dieselbe obere Höhengrenze erreicht wie der Flaumeichenwald und im wesentlichen auch auf dieselben Expositionslagen beschränkt ist. Die sehr unterschiedliche Intensität und Dauer der Sonneneinstrahlung in Abhängigkeit von der Reliefgestaltung muss daher, sofern man eben von den aktuellen Beobachtungen, d. h. von der tatsächlichen Verbreitung der heutigen Q.pubescens - Bestände ausgeht, als entscheidender Faktor für die räumliche Verteilung der Art im Grenzbereich ihres Arealvorkommens gelten.

Geländeklimatologische Untersuchungen (ROTEN 1964; PRIMAULT/CATZEFLIS 1966; BURNAND 1976) unterstreichen nun, dass bei der generellen strahlungsbegünstigten Situation des mittleren Wallis durch die lokale Ausgestaltung des Reliefs, insbesondere bezüglich der Exposition, Situationen bzw. Standorttypen geschaffen werden, die zusammen mit den beobachteten Vorkommen von Q. pubescens die ökologische Wertigkeit der Art abzugrenzen verhelfen. BURNAND (1976) hat für 131 Punkte im mittleren Wallis die Sonneneinstrahlungs summen für die Zeit vom 24. 3. bis 19.10., was zeitlich noch länger ist als die Vegetationsperiode von $\mathrm{Q}$. pubescens, berechnet. Danach zeigen die gesamten nördlichen Talflanken des Haupttales erheblich höhere Einstrahlungssummen als die südlichen Talflanken (Fig. 6). Nur im Uebergangsbereich von nördlich einmündenden Tälern, welche eine
E-W-Exposition und damit eine Reduzierung der direkten täglichen Sonneneinstrahlung bewirken, sind auch auf der Nordseite des Haupttales geringere Summen vorhanden. Die grössten Unterschiede bestehen - wie auf Grund des hier genau W-E angelegten Talverlaufs auch zu erwarten - zwischen der N- und SFlanke des oberen Talverlaufes, wo auf der südlichen Seite die geringsten Werte des gesamten Raumes vorhanden sind. Aber auch im weiteren Verlauf erreichen die linksseitigen Hänge des Rhonetales, obwohl mehr nach NW exponiert, nicht die Werte der Strahlungssummen des rechtsseitigen Talverlaufes. Der Vergleich zwischen der räumlichen Verteilung der errechneten Sonneneinstrahlungssummen und der tatsächlichen Verbreitung von Q. pubescenslässt die folgenden Korrelationen erkennen:

1) Die unteren, S-exponierten Talflanken des nördlichen Mittelwallis mit den grössten Einstrahlungssummen währeng der Vegetationsperiode $\left(156-200 \mathrm{kcal} \mathrm{cm}^{-2}\right)$ besitzen die stärksten Flaumeichenvorkommen im gesamten Untersuchungsbereich und erreichen bis zu $45 \%$ am Gesamtbestand in Föhren/Flaumeichen-Mischwäldern.

2) Die Hänge mit mittleren Einstrahlungssum-

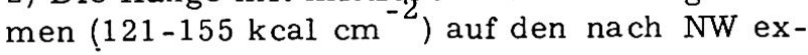
ponierten Talflanken des südlichen Mittelwallis weisen eine wesentlich geringere Vorkommensfrequenz auf, obwohl der Anteil von Q. pubescens in Föhrenwäldern immer noch bis zu $15 \%$ betragen kann.

3) Auf den unmittelbar $\mathrm{N}$-exponierten Hängen des östlichen Teiles des mittleren Wallis mit den geringsten Einstrahlungssummen (100 $120 \mathrm{kcal} \mathrm{cm}^{-2}$ ) werden nur sehr vereinzelt Flaumeichen angetroffen. Ihre geringen Vorkommen rechtfertigen nicht die Ausgliederung eines bestimmten Verbreitungstypes.

Reine Flaumeichenwälder im bestandssoziologischen Sinne des Quercetum pubescentis sind wegen des starken menschlichen Einflusses in der kollinen Stufe selten. Am häufigsten kommt die Art als Strauch oder kleiner Baum in offenen und stark lichtdurchlässigen Föhrenwäldern (Pinetum silvestris) vor.

Eine weitere, aus den Pflanzenbeständen selbst sich ergebende Stütze für die ökologische Standortwertigkeit ist das häufige Vorkommen von Trockenrasen, dürren Schwingelmatten und Federgrasfluren, die unter dem Begriff "Felsensteppe" (FREY 1934) oder "Innerwalliser Steppenrasen" (BRAUN-BLANQUET 1961) zusammengefasst und in Fig. 8 als xerophile Kraut- und Strauchgesellschaften an einer Profillinie kartiert wurden. Auf felsigen Hängen aus Kalk- und Dolomitschiefern mag dieser Vegetationstyp als klimatische Klimax angesehen werden. Der Anteil der Hemikryptophyten und Chamaeophyten - letztere in ihrer Häufigkeit 
durchaus mediterranen Gesellschaften vergleichbar - am Lebensformenspektrum ist auffallend hoch. Es muss in diesem Zusammenhang von besonderer Bedeutung erscheinen, dass der Anteil von mediterranen Arten (Ononis pusilla, Linum tenuifolium, Harniaria glabra, Satureja Acinos u. a. ) und von sarmatischaralokaspischen Steppenpflanzen (Stipa pennata, S. capillata, Artemisia campestris, Poa bulbosa, Euphorbia Seguieriana, Onobrychis viciifolia var. arenaria, Festuca ovina var. glauca, Anemone montana, Galium verum, Silene Otites, u. a. ) sehr gross ist. Diese Steppenrasen sind fast ganz überwiegend auf S-exponierte Lagen des Haupttales konzentriert und häufig in unmittelbarer räumlicher Nachbarschaft zum Pinetum silvestris mit hohem Flaumeichenanteil, häufig sogar in direkter gesellschaftlicher Verbindung in einer einzigen Assoziation ( = Föhren/Flaumeichen-Wald mit hohem Anteil an xerophilen Kräutern und Sträuchern).

1.2. Topographisch bedingte Spätfrostgefahr Es ist von vornherein zu erwarten, dass im zentralen Rhonetal auf Grund der starken Strahlungsgegen sätze - insbesondere auch zwischen Tages- und Nachttemperaturen - eine strahlungsbedingte Spätfrostgefahr zu Beginn der Vegetationsperiode von Quercus pubescens d. h. in der zweiten Aprilhälfte - zumindest potentiell eine standörtliche Einschränkung bedingt. BURNAND (1976) konnte auf Grund von Messungen der Minimumtemperaturen in Strahlungsnächten entlang von acht Profilen durchs Rhonetal nachweisen, dass bis zu einer Höhe von $950 \mathrm{~m}$ - d.h. im wesentlichen in der Stufe maximalen Vorkommens der Flaumeiche die Bildung von Kaltluftseen stark von der topographischen Geländegestaltung abhängig ist und nur in abgeschlossenen Muldenlagen im Bereich der Talsohle auftraten.

Solche Muldenlagen sind charakteristisch für den Pfinwald mit seinem sehr gegliederten Relief im Bergsturzgebiet bei Sierre. Der Pfinwald besteht zum grössten Teil aus einem Föhrenwald mit unterschiedlichem Anteil von Quercus pubescens. Die Flaumeiche kann in der Oberschicht nur auf den Hügeln angetroffen werden (Fig. 9). Abseits dieser Kuppenlagen wird sie nur in der Strauchform angetroffen, meist auch in stark verminderter Frequenz. Sehr auffallend ist nun das Fehlen der Flaumeiche in den ausgesprochenen Muldenlagen des Bergsturzgebietes, wo auch im Unterwuchs sehr arme Föhrenbestände auftreten. BURNAND (1976) erhielt bei seinen zitierten Temperaturmessungen durch das Rhonetal in Strahlungsnächten (Ende April) für drei dieser Mulden Werte von $-1,7^{\circ},-1,4^{\circ}$ und $-1,1^{\circ}$, während die benachbarten Hügel Werte von $+1,2^{\circ},+1,0^{\circ}$ und $0,9^{\circ}$ aufwiesen. Auch wenn der Schwellenwert der Frosttoleranz noch geringfügig tiefer liegen mag als die zi- tierten Werte, so muss doch die Spätfrostgefahr das Vorkommen der kälteempfindlichen Flaumeichen negativ beeinflussen.

Dem entspricht nun aber auch der absolute Grenzverlauf des Vorkommens auf der Höhe von Brig (Fig. 6 und 7), wonach östlich davon - d.h. im oberen Wallis - Flaumeichen nicht mehr vorkommen und auch in der unteren talnahen Stufe zunächst von Föhren und weiter talaufwärts von Fichten und Lärchen ersetzt werden. Das ökologische Klimadiagramm von Brig (Fig. 6) zeigt - im Vergleich zu den westlich gelegeneren Stationen Sierre und Martigny - eine wesentlich stärkere Spätfrostgefährdung nach dem Beginn der Vegetationsperiode von Quercus pubescens. Diese starke Spätfrostgefährdung sowie die Beobachtungen über das aktuelle Verbreitungsmuster der Art im Pfinwald ergeben, dass die Flaumeiche im mitțleren Wallis klimaökologisch an der Grenze ihres Verbreitungsgebietes steht. Wo die Kontinentalität, d. h. vor allem das Auftreten strahlungsbedingter Spätfröste stärker wird wie z. B. im östlich anschliessenden oberen Wallis - kann Q. pubescens sich nicht mehr erfolgreich etablieren und die Vorkommen hören demèntsprechend ganz auf.

1.3. Der Charakter der Niederschlagsverhältnisse während der sommerlichen Vegetationsperiode (insbesondere Juni/Juli) unter besonderer Berücksichtigung der Häufigkeit von Monatssummen und Trockenperioden

Während Expositionslagen und davon abhängige Einstrahlungssummen die standörtliche Verteilung der Flaumeiche innerhalb des Haupttalraumes des mittleren Wallis entsprechend beeinflussen, ergibt sich nun die Frage, warum gerade hier in dieser besonderen topographischen Situation der Zentralalpen eine solche floristisch-quantitativ starke Präsenz der Art vorhanden ist, die in der natürlichen Vegetation - also vor anthropogenem Einfluss nach pollenanalytischen Untersuchungen sogar noch erheblich höher war (MARKGRAF 1969). Es muss in diesem Zusammenhang auch erneut darauf hingewiesen werden, dass das mittlere Wallis immerhin an der Grenze des Arealbereichs von $Q$. pubescens liegt.

Da experimentell-ökologische Transpirationsmessungen von LARCHER (1961) am Gardasee ergeben haben, dass die Flaumeiche eine angespannte Wasserversorgung während der sommerlichen Vegetationsperiode ohne Schaden überstehen kann und damit den meisten mesophilen Laubholzarten Mitteleuropas im Kampf um den Raum konkurrenzüberlegen ist, kann als eine entscheidende Prämisse für die räumliche Verbreitung der Art der Charakter der Niederschlagsverhältnisse während der sommerlichen Vegetationsperiode angesehen werden.

Zunächst weist der Jahresgang der mittleren 
monatlichen Niederschlagssummen für Martigny und Sierre einen insgesamt ausgeglichenen Verlauf auf; er unterscheidet sich jedoch dadurch von Stationen unmittelbar nördlich der Alpen, indem das sommerliche Maximum schon in Martigny sehr stark abgeschwächt ist und nach E zu - ins innere Wallis hinein - an Bedeutung verliert, um in Brig durch ein Sommerminimum ersetzt zu werden.

Die mittlere Zahl der Tage mit Niederschlag $\hat{\jmath} 1,0 \mathrm{~mm}$, aufgeteilt nach Jahreszeiten, zeigt im Haupttalniveau eine deutliche Abnahme von W nach $\mathrm{E}$ (= abnehmende Niederschlagsbereitschaft), was sich insbesondere in der sommerlichen Vegetationsperiode bemerkbar macht (Martigny 29,0 Tage; Sion 25,1 Tage; Visp 20,8 Tage). (Tab. 2)

Von einem ökologisch bedeutsameren Stellenwert ist allerdings die relative Häufigkeit der monatlichen Niederschlagssummen, da durch diese die Wahrscheinlichkeit von bestimmten Niederschlagsmengen auf die sommerliche Vegetationsperiode, d.h. die entscheidende Zeit für einen ausgeglichenen pflanzlichen Wasserhaushalt, abgelesen werden kann. Für drei repräsentative Stationen im Talbereich des mittleren Wallis wurde daher die Häufigkeitsverteilung der monatlichen Mittelwerte nach Mengenklassen bzw. Intervallen, welche sich nach der logarithmischen Skala von SCHNEIDERCARIUS (1955) richten, errechnet: für Martigny ganz am westlichen Rand, wo durch das untere Walliser Rhonetal noch ein "Zugang" zum feuchteren Bereich der nördlichen Alpen gegeben ist; für Sion etwa im Zentrum des Talbereichs und für Visp im östlichen Randbereich (Fig. 10).

Von einer besonderen Bedeutung für die Vegetation sind naturgemäss die Häufigkeitswerte bezüglich bestimmter Niederschlagsklassen während der Sommermonate, d. h. Juni bis August. In unserem Zusammenhang werden insbesondere Juni und Juli beachtet, da ein durch eventuelle Trockenheit bedingter Stress in der pflanzlichen Wasserversorgung im Monat August, d. h. schon im letzten Drittel der Vegetationsperiode, pflanzenphysiologisch nicht unbedingt schädlich sein muss (LARCHER 1961).

Es kann nach BAGNOULS/GAUSSEN (1952) angenommen werden, dass ein Monatsmittel von weniger als $50 \mathrm{~mm}$ Niederschlag bei Mitteltemperaturen von $20^{\circ}-30^{\circ}$ pflanzenphysiologisch den Charakter eines Trockenmonats besitzt, obwohl LAUER (1953) in seiner Kritik zu Recht darauf hinweist, dass eine exakte Definition des Begriffes "Trockenmonat" hierdurch allein nicht erfüllt sein kann.

Innerhalb der Mengenklassen, die insgesamt den grössten Anteil an den Niederschlagssummen besitzen, d. h. von $25 \mathrm{~mm}-99,9 \mathrm{~mm}$, fallen in Martigny und Sion die grössten pro-

\begin{tabular}{|c|c|c|c|c|c|}
\hline Stationen & 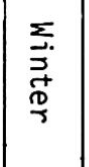 & 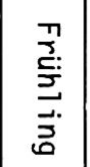 & 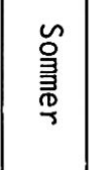 & 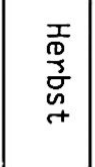 & $\frac{c}{5}$ \\
\hline Haupttalniveau & & & & & \\
\hline Martigny & 23.8 & 25.2 & 29.0 & 24.9 & 102.9 \\
\hline Sion & 20.4 & 20.5 & 25.1 & 21.3 & 87.3 \\
\hline $\begin{array}{l}\text { Visp } \\
\text { Nördl iches Mittelwall is }\end{array}$ & 20.6 & 20.7 & 20.8 & 20.6 & 82.7 \\
\hline Montana & 27.3 & 28.8 & 30.3 & 26.3 & 112.7 \\
\hline Leukerbad & 25.3 & 28.1 & 31.7 & 25.7 & 110.8 \\
\hline $\begin{array}{l}\text { Kippel } \\
\text { Südliches Mittelwallis }\end{array}$ & 27.6 & 29.7 & 31.7 & 25.7 & 115.6 \\
\hline $\operatorname{Nax}$ & 21.5 & 24.1 & 25.7 & 21.7 & 93.0 \\
\hline Vissoye & 20.5 & 23.2 & 25.8 & 21.6 & 91.1 \\
\hline Grächen & 17.5 & 20.3 & 23.2 & 19.7 & 80.7 \\
\hline Zermatt & 18.8 & 24.1 & 27.5 & 22.6 & 93.0 \\
\hline
\end{tabular}

Tab. 2: Mittlere Anzahl der Tage mit Niederschlag $=1.0 \mathrm{~mm}(1901-1940)$ im mittleren Wallis.

zentualen Häufigkeitswerte auf die Sommermonate, was dem schon aus dem Jahresgang der Mittelwerte ersichtlichen Sommermaximum entspricht. Allerdings kann eine bemerkenswerte Differenzierung bezüglich der Mengenklassen, welche notwendigerweise von pflanzenphysiologischer und vegetaionsökologischer Bedeutung sein müssen, zwischen den einzelnen Stationen beobachtet werden. Während in Martigny in der Klasse $50-99,9 \mathrm{~mm}$, welche als pflanzenphysiologisch ausreichend angesehen werden muss, während der Monate Juni und Juli Häufigkeiten von über $50 \%$ erreicht werden und sogar Monatssummen von über $100 \mathrm{~mm}$ mit einer Wahrscheinlichkeit von $8 \%$ auftreten können, ist bei Sion eine Linksverschiebung und gleichzeitige Verminderung der Häufigkeitswerte zu beobachten: in Sion hat sich ein geringerer Spitzenwert von $41-50 \%$ in die Klasse $25-49,9 \mathrm{~mm}$, welche schon den Charakter eines pflanzenphysiologisch wirksamen Trockenmonats repräsentiert, verlagert, während Niederschläge im Juli von mehr als $100 \mathrm{~mm}$ nur noch mit einer Wahrscheinlichkeit von weniger als $2 \%$ auftreten. In Visp sind sogar in den Monaten Ju- 
ni und Juli neben den geringsten Mittelwerten auch Häufigkeitswerte von nur 31-40\% für das Intervall 25-49,9 mm zu beobachten. Niederschläge von mehr als $100 \mathrm{~mm}$ treten im Juli überhaupt nicht mehr auf.

Bei einem Vergleich der drei Stationen lässt sich daher zusammenfassend herausstellen, dass im Haupttal des mittleren Wallis von W nach $E$ eine abnehmende Niederschlagsbereitschaft bei geringeren Monatssummen eintritt. Es muss daher mit einer angespannten pflanzlichen Wasserversorgung während der Sommermonate gerechnet werden.

Dieser pflanzenphysiologisch wirksame Wasserstress wird auch dadurch hervorgehoben, dass die mittlere Dauer der Niederschläge während eines grossen Teils der Vegetationsperiode nur 2 Stunden 14 Minuten beträgt (Fig. 11). Gleichzeitig beträgt die mittlere Sonnenscheindauer in Sion im Juli 262 Stunden, d. h. $63 \%$ der möglichen Sonnenscheindauer (SCHUEPP 1962), bei einem mittleren monatlichen Temperaturmaximum von $31,8^{\circ} \mathrm{C}$ (ebenfalls Juli). Die tageszeitliche Niederschlagsverteilung (Fig. 11)zeigt auf ein Maximum in den frühen Abend- und Nachtstunden, wonach der erwähnte tägliche Stress durch erhöhte Transpiration während der Tagesstunden nicht vermieden werden kann.

Die im ökologischen Klimadiagramm (Fig. 6) sich ergebende sommerliche Trockenperiode kann auch durch einen errechneten Trockenheitsindex bestätigt werden, welcher nach GIACOBBE (1949) und EMBERGER (1955) zwischen pflanzenphysiologisch trockenen und feuchten Wuchsraumbereichen im Mittelmeergebiet unterscheidet:

$\mathrm{P}^{\mathrm{E}}=$ Sommerregenmenge

M = mittleres Maximum im wärmsten Monat

Falls dieser Quotient 7 unterschreitet, ergibt sich nach EMBERGER eine sommerliche Trockenheit, die sich im Habitus der Pflanzen, etwa durch verstärkte Schutzvorrichtungen gegen erhöhte Transpiration, äussert. Im mittleren Wallis ist für alle Stationen östlich von Martigny dieser Quotient kleiner als 7.

Zusätzlich zur Niederschlagsverteilung sind auch Dauer und Häufigkeit von Trockenperioden von einer entscheidenden vegetationsökologischen Wertigkeit. Gewöhnlich wird als Trockenperiode eine Aufeinanderfolge von Tagen mit unbedeutenden oder ausbleibenden Niederschlägen verstanden. Ueber die sehr unterschiedliche Auslegung des Begriffsinhaltes "unbedeutender Niederschlag" existiert eine umfangreiche Diskussion (teilweise Zusammenfassung bei KUHN 1973). Für unsere Zwekke ergibt sich aus dieser bisherigen Diskussion die Definition einer Trockenperiode als einer Anzahl aufeinanderfolgender Tage mit
Niederschlägen, die geringer sind als ein bestimmter Schwellenwert. KUHN (1973) hat als Schwellenwerte für die Berechnung von Trokkenperioden an verschiedenen Stationen der Schweiz 0,2 und $5 \mathrm{~mm}$ täglicher Niederschlag gewählt.

Die Häufigkeitsverteilung von Trockenperioden verschiedener Schwellenwerte zeigt, dass diese in Sion insgesamt sehr häufig sind (Fig. 12). So beträgt die mittlere Häufigkeit von drei aufeinanderfolgenden Tagen ohne Niederschlag (Schwellenwert $0 \mathrm{~mm}$ ) im Sommerhalbjahr 7,2. Bei Benutzung des Schwellenwertes $5 \mathrm{~mm}$ täglicher Niederschlag ergibt sich, dass 20-tägige Trockenperioden etwa in jedem 2. Beobachtungszeitraum (= Sommerhalbjahr) auftreten (zum Vergleich: Basel in jedem 4 . Beobachtungszeitraum, während La-Chaux-deFonds auf der W-Seite des Schweizer Jura eine solch lange Trockenperiode nach dieser Häufigkeitsanalyse überhaupt nicht erlebt). Die von KUHN (1973) unter Benutzung einer Summenhäufigkeitsfunktion gewonnenen wahrscheinlichsten extremen Trockenperioden lassen sich in Kombination mit weiteren signifikanten Klimaeigenschaften für eine vorläufige klimaökologische Differenzierung der wichtigsten Vegetationseinheiten der kollinen und montanen Stufe innerhalb der Schweiz heranziehen (Tab. 4).

Es lassen sich die bisherigen Ergebnisse in folgender Weise zusammenfassen, wobei die vegetationsökologische Individualität des Unter suchungsraumes hervorgehoben werden soll: Die Kombination von 1) eindeutiger Tendenz zum jahreszeitlichen Minimum der Niederschlagsverteilung in den Sommermonaten mit gleichzeitiger nach $\mathrm{E}$ abnehmender Niederschlagsbereitschaft bei geringeren Monatssummen von kurzer zeitlicher Dauer, 2) hoher tatsächlicher und relativer Sonnenscheindauer, 3) einer relativ grossen Wahrscheinlichkeit auch länger anhaltender sommerlicher Trokkenperioden und 4) nach E steigender Spätfrostgefahr lassen das mittlere Wallis als einen Raum erscheinen, dessen klimaökologische und pflanzenphysiologisch wirksame Amplitude eine ganz spezifische Vermischung submediterraner und subkontinentaler Eigenschaften aufweist, wie sie vielleicht in einmaliger Weise nur in einem solchen Tal innerhalb der Zentralalpen bei schon vergleichsweise weit nach $\mathrm{S}$ vorgeschobener Breitenlage möglich ist.

Eine entscheidende - auch sicherlich methodisch für andere Raumeinheiten mit unterschiedlichen waldbildenden Florenelementen wichtige - Frage lautet nun: Wie lassen sich die hier analysierten Klimaelemente mit den starken Vorkommen der Flaumeiche in räumlich-standörtlicher und öko-physiologisch sinnvoller Weise vereinigen? Hier muss in erster 
Linie das gegenseitige Konkurrenzverhalten der für einen Raum potentiell zu Verfügung stehenden Baumarten genannt werden, welches insbesondere durch einen überregionalen Vergleich zu gesicherten Ergebnissen führen kann und im Abschnitt IV behandelt wird.

2. Die Föhrenbestände - Verbreitung und standörtliche Assoziationstypen

Die Föhre (Pinus silvestris) ist im gesamten Mittelwallis an den Hängen des Haupttales sowie im unteren, dem Haupttal zugekehrten Bereich der Nebentäler - teilweise auch noch bis in den mittleren Bereich der Nebentäler reichend (Vispertal) - die dominante Baumart bis zu einer Höhe von $1000 \mathrm{~m}$ bis $1500 \mathrm{~m}$. Ihre absolute obere Verbreitungsgrenze liegt in $\mathrm{N}$ Expositionen bei $1700 \mathrm{~m}$, in S-Expositionen bei $2200 \mathrm{~m}$ und kann hier stellenweise die Waldgrenze erreichen (Fig. 4). Die grösste Artmächtigkeit wird jedoch unterhalb von $1200 \mathrm{~m}$ erreicht, wo P. silvestris im gesamten Untersuchungsbereich bestandsbildend auftritt und auf $\mathrm{N}$-exponierten Hängen unterhalb von $700 \mathrm{~m}$ sogar über $80 \%$ der.vorhandenen Baumarten ausmacht (Fig. 3). Auf S-exponierten Hängen bildet die Föhre auch überall den Bestandsschluss; allerdings tritt hier die Flaumeiche im Unterwuchs stärker in Erscheinung. Mit zunehmender Höhe treten vor allem Lärche (Larix decidua) und Fichte (Picea excelsa) in stärkerem Umfange hinzu und ersetzen allmählich die Vorherrschaft der Föhre, wobei soziologisch der Uebergang vom Pinetum etwa zum Piceetum sich zwischen $1000 \mathrm{~m}$ und $1400 \mathrm{~m}$ vollzieht.

Eine räumliche und zugleich ökologisch wichtige Differenzierung kann in unterschiedlichen Bestandszusammensetzungen, d. h. Assoziationstypen gesehen werden.

Eine besonders auffallende und gut ausgeprägte Assoziation wird durch die häufigen, den Boden meist geschlossen bedeckenden Vorkommen von Erica carnea gebildet, welche von

SCHMID (1936) als Pinetum silvestris ericosum bezeichnet und von BRAUN-BLANQUET $\overline{(1961)}$ als Ericeto-Pinetum dem Erico-PinionVerband zugeordnet wird. Der standörtliche Verbreitungsschwerpunkt im mittleren Wallis liegt auf den $\mathrm{N}$-exponierten Hängen, insbesondere im linken Rhonetal. Auffallend ist das starke Zurücktreten der Flaumeiche. Hieraus, vor allem aber auch durch das stärkere Auf treten feuchtigkeitsliebender Kräuter sowie Moosen, kann ein mesophiler Charakter der Gesellschaft abgeleitet werden. Dies würde auch durch das generelle Verbreitungsmuster der Assoziation bestätigt, deren Schwerpunkt nach SCHMID (1936) in den nördlichen und östlichen Alpen liegt.

Auf den $\mathrm{S}$-exponierten Hängen, insbesondere auf den ausgedehnten Hängen des nördlichen Rhonehaupttales kommt eine artenreichere As- soziation vor, welche durch eine Reihe von submediterranen und pontisch-aralokaspischen Arten gekennzeichnet ist. Sie wird von SCHMID (1936) als Pinetum silvestris astragalosum bezeichnet und von BRAUN-BLANQUET (1961) dem trocken-heissen Ononido-Pinion - Verband zugeordnet. Es ist wichtig, dass hie $r$ der Anteil von Quercus pubescens erheblich höher ist, was zweifellos dem xerischen Charakter dieser Gesells chaft entspricht. Auch kann eine stärkere edaphische Beziehung festgestellt werden, da diese Gesellschaft häufig - wenn auch nicht ausschliesslich - auf flachgründigen Kalkrohbö den angetroffen wird. Die räumliche Na chbar schaft $\mathrm{zu}$ den in 1.1. beschriebenen xerophilen Kraut-und Strauchgesellschaften muss als weiterer ökologisch-standörtlicher Parameter gewertet werden, wie überhaupt eine ähnliche Abhängigkeit von den hohen Einstrahlungssummen erwartet werden darf, welche schon die starken Flaumeichenvorkommen auf S-exponierten Hängen begünstigte. Die lockere Baums chicht, die vielfach nicht mehr als $70-80 \%$ des möglichen Kronenraumes beansprucht, lässt viel Licht durchfallen, was das Eindringen von wärmeliebenden Arten in der Strauch-und Krautschicht erleichtert (umfassende Artentabellen bei SCHMID 1936). Die bei der Verbreitung der Flaumeiche abgeleiteten Eigenschaften des Strahlungshaushaltes, der Häufigkeit monatlicher Niederschlagssummen während des Sommers und der Frequenz von Trockenperioden im Sommerhalbjahr können ebenfalls zur Erklärung der starken Frequenz der Föhre benutzt werden, wobei wiederum die Konkurrenzverhältnisse unter den zu Verfügung stehenden Baumarten (= "Artenstock") des euroäischen Florengebietes entscheidend sind. Dies wird im Vergleich mit anderen europäischen Kiefernvorkommen auf eine methodisch sichere Grundlage gestellt werden (Kap. IV, 3).

3. Die Vorkommen der Weisstanne in Beziehung zur Häufigkeit monatlicher Niederschlagssummen grösser $100 \mathrm{~mm}$ (Juni/ Juli)

Die folgenden beobachteten Gesetzmässigkeiten aus dem Verbreitungsmuster der Weisstanne (Abies alba) im mittleren Wallis (Fig. 3) sind für die Diskussion der ökologischen Standortwertigkeit von Bedeutung:

1) Das Verbreitungsgebiet von A. alba ist im wesentlichen auf die montane und subalpine Stufe des nördlichen Mittelwallis beschränkt, wobei die Vorkommen westlich von Brig, also noch vor Beginn des eigentlichen oberen Wallis, aufhören.

2) In den nördlichen Seitentälern tritt die Weisstanne zwischen $1100 \mathrm{~m}$ und $1900 \mathrm{~m}$ als ein regelmässiger Bestandteil der Wälder auf, wobei sie in unterschiedlichem Mengenanteil mit Pinus silvestris, Larix decidua und Picea excelsa vergesellschaftet sein kann. 
Nördliches Mittelwallis mit Vorkommen von Abies alba

\begin{tabular}{|c|c|c|c|c|c|c|c|c|c|c|c|c|c|c|c|c|c|}
\hline 1 Montana (1453m) & 76 & 60 & 70 & 66 & 62 & 64 & 68 & 76 & 66 & 74 & 67 & 91 & 227 & 198 & 208 & 207 & 840 \\
\hline 2 Leukerbad $(139 \mathrm{~m})$ & 101 & 64 & 75 & 70 & 70 & 92 & 95 & 106 & 78 & 81 & 89 & 107 & 272 & 215 & 293 & 248 & 1028 \\
\hline 3 Kippel (1376m) & 88 & 67 & 86 & 78 & 68 & 66 & 75 & 76 & 67 & 83 & 80 & 102 & 257 & 232 & 217 & 230 & 936 \\
\hline
\end{tabular}

Südliches Mittelwallis ohne Vorkommen von Abies alba

\begin{tabular}{|c|c|c|c|c|c|c|c|c|c|c|c|c|c|c|c|c|c|}
\hline & $\mathrm{J}$ & $\mathrm{F}$ & M & A & M & $\mathrm{J}$ & $\mathrm{J}$ & A & $S$ & 0 & $\mathrm{~N}$ & D & & $\infty$ & & & \\
\hline $4 \mathrm{Nax}(1300 \mathrm{~m})$ & 49 & 46 & 51 & 49 & 48 & 52 & 61 & 67 & 54 & 60 & 53 & 66 & 161 & 148 & 180 & 167 & 656 \\
\hline 5 Vissoye $(1260 \mathrm{~m})$ & 41 & 35 & 46 & 44 & 50 & 60 & 71 & 70 & 48 & 55 & 45 & 52 & 128 & 140 & 201 & 148 & 617 \\
\hline 6 Grächen $(1629 \mathrm{~m})$ & 35 & 31 & 47 & 44 & 56 & 48 & 48 & 52 & 50 & 63 & 41 & 47 & 113 & 147 & 148 & 154 & 562 \\
\hline 7 Zermatt $(1610 \mathrm{~m})$. & 46 & 42 & 50 & 57 & 65 & 65 & 58 & 69 & 60 & 74 & 54 & 54 & 142 & 172 & 192 & 188 & 694 \\
\hline
\end{tabular}

Tab. 3:

Die Verteilung der monatlichen Niederschlagsmengen in der montanen Stufe des nördlichen und südlichen Mittelwallis

3) Es besteht ein deutlicher Unterschied bezüglich höhenabhängiger Vorkommensfrequenz zwischen dem westlichen und östlichen Teil des nördlichen Mittelwallis, der in den folgenden Punkten zusammengefasst werden kann:

a) Im westlichen Teil (Vallée de la Lizerne) bestehen keine sehr grossen Unterschiede der Häufigkeitsverteilung innerhalb der gegebenen vertikalen Amplitude (1100 m - $1800 \mathrm{~m})$. Die relativ grösseren Mengenanteile am Gesamtbestand werden allerdings unterhalb von $1400 \mathrm{~m}$ angetroffen.

b) Im östlichen Teil (Lötschental) werden die grösseren Mengenanteile am Gesamtbestand oberhalb von $1400 \mathrm{~m}$ angetroffen.

c) Aus a) und b) ergibt sich eine stetig höher reichende Konzentration der Häufigkeitsverteilung zwischen dem westlichen und östli chen Bereich der untersuchten Vorkommen. Wenn man daher die von HAINARD (1969) für das Wallis angenommene mittlere Meereshöhe der Weisstanne von $1450 \mathrm{~m}$ als Bezugspunkt nimmt und gleichzeitig eine Zunahme des Niederschlages mit der Höhe zugrunde legt (BOUET 1965), würde dies bedeuten, dass A. alba nach $\mathrm{E}$ $z u$ in ein feuchteres Höhenstockwerk ausweicht oder zumindest den Häufigkeitsschwerpunkt dorthin verlegt.

4) Im westlichsten Nebental (Vallée de la Lizerne) tritt A. alba häufig vergesellschaftet mit der Rotbuche (Fagus silvatica) auf, welche hier ihre Grenze gegen die Zentralalpen erreicht und die auf Grund ihrer bekannten Standortansprüche (ELLENBERG 1963) auf einen gleichmässigen Jahresniederschlag angewiesen ist.

Das Verbreitungsmuster der Weisstanne kann nun tatsächlich mit charakteristischen, räumlich differenzierten Eigenschaften der Niederschlagsverhältnisse korreliert werden. Zunächst sind die mittleren monatlichen Niederschalgsmengen im Sommer in der montanen Stufe des nördlichen Mittelwallis, d.h. im Verbreitungs gebiet der Weisstanne, mit über $90 \mathrm{~mm}$ erheblich höher als im südlichen Mittelwallis, wo lokal (Grächen) Werte von weniger als $50 \mathrm{~mm}$ registriert werden (Tab. 3).

Oekologisch wichtig ist auch hier wieder die wahrscheinliche Häufigkeit von Niederschlagssummen im Juni/Juli: sie beträgt im nördlichen Mittelwallis (Station Leukerbad, $1391 \mathrm{~m}$ ) für Niederschlagsmengen grösser $100 \mathrm{~mm}$ zwischen $41 \%$ und $50 \%$, während sie im südlichen Mittelwallis (Station Zermatt, $1610 \mathrm{~m}$ ) für die gleiche Mengenklasse kleiner $10 \%$ beträgt (Fig. 13).

Die Verbreitung der Weisstanne und die Häufigkeitsanalysen sommerlicher Niederschlagsmengen ergeben eine deutliche Differenzierung in ein niederschlagsreicheres, auch im Sommer feuchteres nördliches Mittelwallis und ein niederschlagsärmeres, sommertrockeneres südliches Mittelwallis.

Die aus den regionalen Analysen innerhalb des nördlichen Mittelwallis (Fig. 3) erkennbare Tatsache, nach der sich von $W$ nach $E$ eine stetig 
höher reichende Konzentration der Haufigkeitsverteilung der Weisstanne ergibt, lässt sich mit dem zur Verfügung stehenden Material der einzelnen Stationen nicht erklären. Allerdings weist ZENTGRAF (1949/50) für die Vorkommen der Weisstanne im süddeutschen Raum darauf hin, dass neben der Niederschlagshöhe auch die Höhe der relativen Luftfeuchtigkeit für das Konkurrenzverhalten von A. alba entscheidend sein könnte. Es kann daher vermutet werden, dass sich das luftfeuchtere Stockwerk über dem relativ trockenen Walliser Haupttal von W nach $E$ anhebt bzw. feuchtere Luft aus dem Raum nördlich der Alpen im W unseres Untersuchungsgebietes topographisch tiefer hinabreicht als nach den Zentralalpen zu. Die Vorkommen der sehr auf Luftfeuchtigkeit angewiesenen Rotbuche im westlichsten Teil (Vallée de la Lizerne) kann als ein weiterer Indikator hierzu angesehen werden.

Demnach erscheinen als begrenzende Faktoren des Verbreitungsareals der Weisstanne mit zunehmender Höhe der Mangel an Sommerwärme, wobei freilich die Konkurrenzüberlegenheit der toleranzfähigeren Fichte (Picea abies) eine wichtige Rolle spielt. Sommertrockenheit muss dagegen als die entscheidende Ursache für die Arealeinschränkung nach den Zentralalpen gewertet werden.

IV, Ueberregionaler Vergleich mit weiteren charakteristischen Vorkommensbereichen in Europa

1. Konkurrenzauslese und klimaökologische Beziehungen unter besonderer Berücksichtigung der kollinen und montanen Stufe der nördlichen und zentralen Schweiz

Es wurde schon darauf hingewiesen, dass das gegenseitige Konkurrenzverhalten der für einen Raum auf Grund der jeweiligen Arealgestaltung potentiell zur Verfügung stehenden Arten von entscheidender Bedeutung ist. Dieses Konkurrenzverhalten wird - neben der edaphischen Situation - zu einem grossen Teil von klimaökologischen Einflüssen gesteuert.

Die überwiegende Mehrheit aller Laubholzarten, welche auf Grund ihrer Arealgestaltung für das mittlere Wallis in Frage kommen, erweist sich unter den gegebenen klimatischen Bedingungen als konkurrenzunterlegen. Dies gilt zunächst für die mesophilen Arten des mitteleuropäischen Fagion-Verbandes (Fagus silvatica), Quercus petraea, Q. robur, $\overline{\text { Car- }}$ pinus betulus, Fraxinus excelsior, Acer pseudoplatanus, A.platanoides, Tilia cordata, T. platyphyllos, Ulmus glabra, U. laevis), die keine der Flaumeiche gleichwertigen Schutzvorrichtungen gegen erhöhte sommerliche Transpiration entwickelt haben 2). Eine Kon- kurrenzunterlegenheit ergibt sich aber auch für die immergrünen Laubholzarten des eumediterranen Quercion - Verbandes, da einerseits im Wallis eine winterliche Unterbrechung der Vegetationszeit vorhanden ist, andererseits aber bei diesen Arten, besonders der Steineiche (Quercus ilex), ein grosser Teil des Assimilationsertrages in den Aufbau der besonderen, den Transpirationsverlust hemmenden Schutzgewebe eingesetzt wird, so dass dieser Teil für das eigentliche Wachstum, welches im Wettbewerb um den Raum wichtig ist, nicht zur Verfügung steht.

Es ist naturgemäss kaum möglich, eine exakte "messbare" Beziehung zwischen dem für die Bestandsbildung und damit für den physiognomischen Charakter der Vegetation entscheidenden Konkurrenzverhalten der verschiedenen Baumarten und den jeweils vorherrschenden regionalklimatischen Gegebenheiten herzustellen.

Der Vergleich verschiedener Waldtypen mit unterschiedlichem Anteil insbesondere an $\mathrm{Bu}$ chen (F. silvatica) und Eichen (Q. robur, Q. petraea, Q. pubescens) mit ökologisch signifikanten Klimaeigenschaften vermag jedoch deutliche Uebereinstimmungen zu zeigen (Tab.4). Es wird in unserem Zusammenhang die mittlere sommerliche Niederschlagsmenge $\left(\mathrm{P}^{\mathrm{E}}\right)$ mit dem mittleren Maximum der Temperatur im Juli $\left(\mathrm{mT}^{\mathrm{J}}\right)$ in Beziehung gebracht. Mit abnehmendem Quotienten $\mathrm{P}^{\mathrm{E}} / \mathrm{mT}^{\mathrm{J}}$ lässt sich in der natürlichen Waldvegetation eine eindeutige Abnahme des Rotbuchenanteils mit gleichzeitig gesteigertem Vorkommen von Eichen beobachten, zunächst allerdings nur von Traubeneiche (Q. petraea) und Stieleiche (Q. robur) ohne bedeutendere Vorkommen von Flaumeichen. Natürliche Eichen/Hainbuchen-Wälder, in denen die Rotbuche eine geringe Rolle spielt oder so gar fehlt, sind denn auch tatsächlich aus vielen Bereichen Mitteleuropas bekannt. Erst wenn der Quotient einen kritischen Wert von 7 erreicht, treten Flaumeichen mit Föhren in grossem Umfang hervor.

Eine bezeichnende ökologische Parallelität besteht in der gleichzeitigen Zunahme der länger andauernden extremen sommerlichen Trockenperioden - begrenzt durch $5 \mathrm{~mm}$ täglicher Niederschlag -, welche sich von 15,34 Tagen in der Traubeneichen/Buchenwaldregion des Randbereiches der nördlichen Alpen (Station Altdorf) um mehr als das Doppelte bis auf 32,62 Tage in der Föhren/Flaumeichenregion des mittleren Wallis (Station Sion) steigert. Es ergibt sich hieraus, dass die Abnahme des Quotienten. $\mathrm{P}^{\mathrm{E}} / \mathrm{mT}^{\mathrm{J}}$ und die gleichzeitige $\mathrm{Zu}$ nahme der Dauer der häufigsten extremen Trockenperiode im Sommerhalbjahr als klimaökologischer Gradient für den Uebergang von feuchtigkeitsliebenden rotbuchenreichen Waldgesellschaften über Eichen/Hainbuchenwälder 


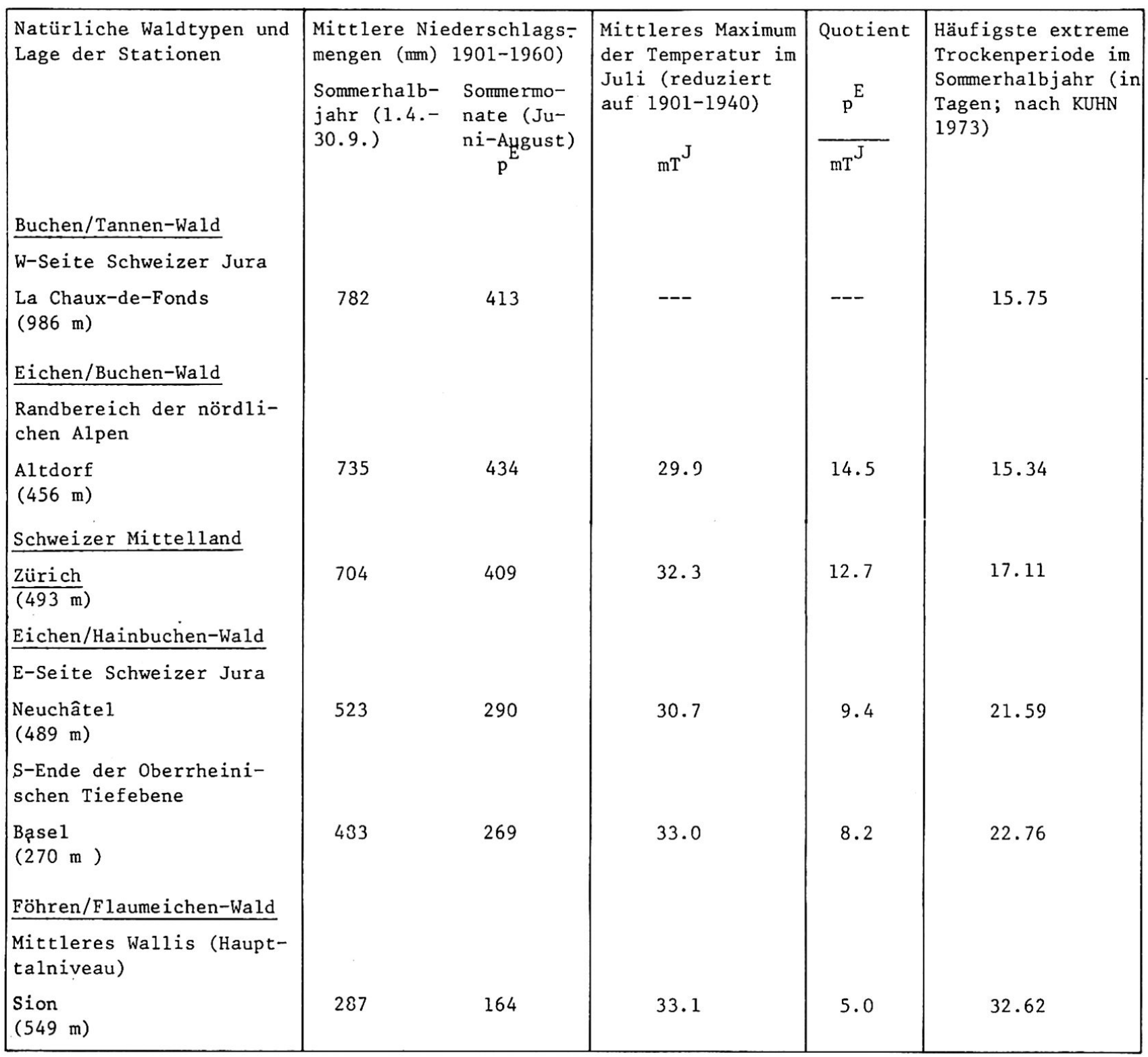

Tab. 4: Oekologisch signifikante Klimaeigenschaften in Beziehung zu den Hauptwaldtypen der kollinen und montanen Stufe der nördlichen und zentralen Schweiz

zu den xerischen Föhren/Flau meichenwäldern gewertet werden muss.

In räumlicher Hinsicht ist dies nicht ein einfaches N-S- oder W-E-Gefälle, sondern in entscheidendem Masse von der topographischen Situation und der Ausbildung des Reliefs abhängig. Basel am S-Ende der Oberrheinischen Tiefebene in der natürlichen Vegetationseinheit des Eichen/Hainbuchenwaldes weist mit 22,76 Tagen für die häufigste extreme Trockenperiode im Sommerhalbjahr einen erheblich höheren Wert auf als Altdorf in der kollinen Stufe der nördlichen Alpen. Auch Luv- und Leeseiten können auf kurze Horizontalentfernung sehr stark modifizieren, wie das Beispiel des Schweizer Jura zeigt: Tannen/Buchenwald auf der WSeite mit 15,75 Tagen für die häufigste extreme
Trockenperiode (Station La Chaux-de-Fonds, $986 \mathrm{~m}$ ) 3) und Eichen/Hainbuchenwald auf der E-Seite mit 21,59 Tagen für die häufigste extreme Trockenperiode (Station Neuchatel, $489 \mathrm{~m})$.

2. Die europäischen Flaumeichenvorkommen submediterrane und subkontinentale Gradienten

Die ökologisch-räumlichen Beziehungen von Quercus pubescens innerhalb Europas zeigen deutlich die Wertigkeit der Art zwischen der mitteleuropäisch-mesophilen Fallaub- und der eumediterranen Hartlaubregion (Fig. 14). Die in Fig. 14 benutzten Pfeile bedeuten dabei nicht eine florengeschichtlich bedingte Ausbreitungsrichtung, obwohl sie im Falle von Q. pubescens und Pinus silvestris teilweise damit überein- 
stimmen. Sie gelten vielmehr als Darstellung von ökologischen Gradienten, die bei der gross räumigen Arealgestaltung für die Verbreitung und Integration der hier behandelten Baumarten in die bestehenden Vegetationseinheiten und Pflanzengemeinschaften verantwortlich sind.

Danach liegt das Schwergewicht der Verbreitung, von dem aus die Art an ökologischen Gradienten zur Grenze ihres Vorkommensspektrums vordringt, in der montanen bzw. submontanen Stufe der humiden Höhenstufenfolge (sensu WALTER 1968) des nördlichen Mittelmeergebietes. Dies wird insbesondere dadurch hervorgehoben, dass Q. pubescens in dieser Stufe an einem W-E-Profil im gesamten Mittelmeerraum erscheinen würde, vom nördlichen Teil der Iberischen Halbinsel über die gesamte Apenninenhalbinsel bis zu den nordanatolischen Randgebirgen und zur Krim (KUEMMEL 1948). Die ökologischen Grenzbedingungen im räumlich wirksamen Kampf mit der immergrünen Steineiche (Q.ilex), die das unterste Stockwerk einnimmt, sind eine temperaturbedingte winterliche Unterbrechung der Vegetationszeit und mit der Höhe zunehmende Niederschläge auch während der sommerlichen Vegetationsperiode. Bei abnehmendem Niederschlag innerhalb derselben Höhenstufe können Flaumeichenbestände durch immergrüne $\mathrm{Na}$ delholzgesellschaften, häufig von Pinus pina ster und P. halepensis, ersetzt werden. Das Verbreitungsmuster von Q. pubescens vom Mediterrangebiet im engeren Sinne nach $\mathrm{N}$ zu ist in sich sehr komplex und wurde häufig im Zusammenhang mit der Abgrenzung der immergrünen mediterranen Vegetation gesehen (RIKLI 1943; OBERDORFER 1948; MARKGRAF 1952; HORVAT 1962). Für den hier ausgeschiedenen submediterranen Gradienten ist zunächst - ähnlich wie in der humiden Höhenstufenfolge im vollmediterranen Bereich beim Uebergang der entsprechenden immergrünen zur sommergrünen Vegetation die temperaturbedingte winterliche Unterbrechung der Vegetationszeit sowie die Zunahme der sommerlichen Niederschläge entscheidend, welche die Flaumeiche im Kampf um den Raum der immergrünen $Q$. ilex konkurrenzüberlegen erweist. In grosser flächenhafter Ausdehnung mit vielfältigen räumlichen Verzahnungen kann dieser Vorgang in S-Frankreich beobachtet werden (BRAUN-BLANQUET et al. 1952; FREITAG 1964). Es wird gerade hier sehr deutlich, dass das floristisch gut zu fassende Quercetum pubescentis sich als Klimaxwald zwischen das Quercion ilicis - bzw. seiner südfranzösischen Ausprägung des Quercetum ilicis galloprovinciale - und den mitteleuropäischen Fagion - Verband, d. h. die nördlich anschliessende Buchenwaldregion, schiebt (KUEMMEL 1948).
Nach der ökologischen Klimaklassifikation von WALTER/LIETH (1967) gehören die meisten der südfranzösischen Stationen im Gebiet des Fla umeichenwaldes (zur genauen Abgrenzung vgl. FLAHAULT 1937) zum submediterranen Klimabereich, in welchem die Dürrezeit durch eine Trockenzeit ersetzt wird. Die Niederschläge sind insgesamt jedoch geringer als in den Gebirgsstationen der Apenninenhalbinsel.

Der Flaumeichenwald verliert nach $\mathrm{N}$ zu sein geschlossenes Areal, um zwischen Nancy und Strassburg die absolute Verbreitungsgrenze zu erreichen. In regionalklimatisch günstigen $\mathrm{Be}-$ reichen - wie z.B. bei Colmar am E-Fuss der Vogesen mit Ausprägung einer sommerlichen Trockenzeit (vgl. Klimadiagramm Colmar bei WALTER/LIETH 1967) -, wo öko-physiologisch dieselben Zusammenhänge vermutet werden können wie im eigentlich submediterranen Bereich, können Flaumeichenwälder z.T. in grösserer Ausdehnung angetroffen werden. Es ist in diesem Zusammenhang wichtig, dass die Flaumeiche im räumlichen Kampf mit anderen Laubholzarten neben der Bevorzugung strahlungsbegünstigter Expositionslagen auf edaphisch trockene Standorte (Kalkstein) verdrängt wird, während andere Arten in strahlungsschwächeren Expositionslagen bei zunehmender Bodenfeuchte und -versauerung konkurrenzüberlegen sind (Fig. 15). Das für viele Pflanzenarten gültige Gesetz der relativen Standortkonstanz (ELLENBERG 1 $\overline{963 \text { ) wird }}$ hierdurch bestätigt.

Ein bisher als solcher nicht herausgestellter Gradient richtet sich von der mediterranen Zone aber auch zu subkontinentalen Klimaverhältnissen, wie sie in weiten Bereichen der Balkanhalbinsel angetroffen werden. Während auch hier - klimaökologisch gesehen - submediterrane Flaumeichenwälder vorhanden sind - vor allem im S des Gebietes (vgl. Vegetationskarte von HORVAT 1962) -, sind Flaumeichen aber auch in der östlichen sehr viel ausgedehnteren serbisch-bulgarisch-westmazedonischen Zerreichenregion (Quercion confertae) vorhanden, welche floristisch sehr starke Beziehungen zu den kontinental-xerophilen russischen Steppengebieten besitzt. Die Klimadiagramme für diesen Raum (nach WALTER/ LIETH) weisen teilweise niedrigere Niederschlagsmengen auf als sonst im nördlichen Mittelmeergebiet, wobei das jahreszeitliche Maximum häufig in den Frühsommer fällt. Insgesamt können die Flaumeichenvorkommen im mittleren Wallis durch den hier abgeleiteten und ökologisch erweiterten Vergleich mit den europäischen Vorkommen schärfer abgegrenzt werden. Dabei lassen sich zusammenfassend die folgenden Ergebnisse herausstellen, die ihrerseits wiederum den individuellen Raumcharakter der Haupttalstufe des mittleren Wallis innerhalb der Alpen herausstellen. 
1) die klimaökologisch entscheidenden Voraus setzungen für die Vorkommen von Q. pubescens im mittleren Wallis können als eine Ueberlagerung von submediterranen und subkontinentalen Faktoren bezeichnet werden (vgl. Kap. III, $1)$.

2) Die klimaökologische Grenze im Wallis wird durch den subkontinentalen Gradienten gegeben, d. h. durch das regelmässige Auftreten von Spätfrösten.

3) Die Ueberlagerung von submediterranen und subkontinentalen Faktoren lässt im Grenzbereich des Vorkommens von Q. pubescens eine besonders starke Vorkommenshäufigkeit hervortreten.

3. Die postglaziale Entwicklung der Arealgestaltung von Föhren in ihren ökologischen Beziehungen zum heutigen Verbreitungsmuster

Die ökologische Stellung der konkurrenzschwachen Föhre lässt sich am ehesten durch einen Vergleich der postglazialen Vegetationsentwicklung darstellen, wobei wiederum das zentrale Wallis Ausgangs- und Beziehungsraum sein soll (Fig. 16)

Als Ergebnis der heute erkennbaren und durch Pollendiagramme abgesicherten spät- und postglazialen Vegetationsentwicklung im Wallis (MARKGRAF 1969) lassen sich für die Diskussion über die rezente ökologische Standortwertigkeit die folgenden grundsätzlichen Leitmerkmale herausstellen.

Die Föhre konnte von Beginn der spätglazialen Vegetationsentwicklung bis zur Jetztzeit ihre Dominanz in der von ihr eingenommenen Höhenstufe - mit Schwerpunkt von $700 \mathrm{~m}$ bis $1200 \mathrm{~m}$ - erhalten. Dabei ist ihr frühes Erscheinen im Alleröd als Erstbesiedler auf den lange Zeit vom Eis bedeckten Rohböden und/ oder Tundrenböden durch ihre weite ökologische Amplitude und der damit verbundenen hohen Toleranzkraft sowie Anspruchslosigkeit hinreichend begründet und steht im Einklang mit der spät- und postglazialen Vegetationsentwicklung Mitteleuropas (FIRBAS 1949). Ueberraschend muss jedoch die relative Arealkonstanz bis zum Subatlantikum erscheinen, welche eine signifikante Abweichung von den mitteleuropäischen Verhältnissen nördlich der Alpen bedeutet, wo im Postglazial die Föhre mehr und mehr von ihrem ursprünglich ebenfalls grossen Verbreitungsgebiet auf echte Reliktstandorte verdrängt wurde. Die Gründe für die weitgehende Arealkonstanz der Föhre, die nur unterhalb von $800 \mathrm{~m}$ zugunsten der Flaumeiche sowie zwischen $1200 \mathrm{~m}$ und $1400 \mathrm{~m} \mathrm{zu}-$ gunsten der Weisstanne und Fichte gegenüber der ursprünglichen Ausdehnung am Beginn des Postglazials (Subboreal) eingeschränkt wurde - ohne aber die Präsenz der Art vollständig zu verlieren (Fig.15) -, lassen sich daher am ehesten durch einen Vergleich mit der Entwicklung nördlich der Alpen herausstellen.

Die Föhre konnte sich seit Beginn des Postglazials über weite Gebiete Mitteleuropas ausdehnen und erreichte zu Beginn der frühen Wärmezeit (Boreal) ihre grösste räumliche Ausdehnung (vgl. Verbreitungskarte bei FIRBAS 1949), wobei sie zugleich bestandsbestimmend war (über $75 \%$ des Pollenniederschlags nach FIRBAS 1949). Schon im Verlauf der frühen Wärmezeit kam es zu einem spürbaren Rückgang der Föhre (RUDOLPH 1930). Es seien in diesem Zusammenhang die wichtigsten Ueberlegungen herausgestellt, die entsprechend der Methode unseres Vorgehens - gerade bei der Beurteilung der für das Wallis unterschiedlichen Situation die standörtlich-ökologischen Beziehungen betonen. Danach kann der nacheiszeitliche Föhrenrückzug auf folgende Ursachen zurückgeführt werden:

1) Der Wettbewerb mit anderen Holzarten, insbesondere Laubhölzern des Eichenmischwaldes hat die Verbreitung der Föhre konkurrenzschwache Rückzugsgebiete eingeschrïnkt. Dabei waren es immer die bodenarmen Landschaften, vor allem solche mit überwiegenden Sandböden, welche die Ausbreitung anderer Arten schwächten und dadurch die Erhaltung der Föhre begünstigten. Dies ist auf Grund der Pollenwerte für das nordböhmische Kreidesandsteingebiet ebenso festgestellt worden wie für die Diluvialsande Brandenburgs und Masurens sowie für das fränkische Keupergebiet (FIRBAS 1949).

2) Die nur schwache Präsenz der Kiefer auf den ausgedehnten Sandböden des nordwestdeutschen Tieflandes - im Gegensatz zu den oben erwähnten kiefernreichen Gebieten des Ostens - macht deutlich, dass für den Kiefernrückzug auch klimatische Gründe verantwortlich sein müssen. Dabei ist denn auch stets die relative Niederschlagsarmut der östlichen Erhaltungsgebiete betont worden (RUDOLPH 1930; FIRBAS 1949). Daneben scheint auch der Rückgang der thermischen Kontinentalität seit dem Boreal die Arealeinschränkung begünstigt zu haben, vor allem die Abnahme der Winterkälte, zu der es in den von der Litorinasenkung betroffenen Landschaften um die Nordsee kam. Es lässt sich nun die ökologische Wertigkeit der Föhre auf Grund des räumlich erweiterten Vergleichs für das zentrale Wallis schärfer fassen. Dabei ist zunächst von grundlegender Bedeutung, dass es - mit Ausnahme des artenarmen Flaumeichenwaldes unterhalb von $800 \mathrm{~m}$ - keinen vergleichbaren Laubmischwald im Wallis gegeben hat und damit ein sehr entscheidender Konkurrenzfaktor ausgeschaltet war. Denn die 
sehr frostharte Arve (Pinus cembra) war seit dem Alleröd stets auf die subalpine Stufe beschränkt, und auch die im Boreal eingewanderte Lärche (Larix decidua) konnte als sehr lichtbedürftige Holzart kaum eine ernst hafte Konkurrenz für die Föhre bedeuten, zumal sie auch wegen weitgehender Frostunempfindlichkeit auf die oberen Stockwerke aus weichen konnte. Nur die Weisstanne an der oberen Grenze der Föhrenstufe sowie die Flaumeiche an deren unterer Grenze waren imstande, das Areal von Pinus silvestris einzuengen, ohne jedoch die immer noch grosse Ausdehnung zu gefährden. Die Weisstanne konnte wohl auf Grund ihrer engen ökologischen Amplitude, vor allem des hohen Feuchtigkeitsanspruches, kaum tiefer in das trockenere Haupttal folgen, während die Flaumeiche nur unterhalb von $800 \mathrm{~m}$ optimale Standortbedingungen (= maximale Einstrahlung bei geringer Spätfrostgefährdung) fand.

Die ausgedehnten Föhrenbestände im zentralen Wallis sind daher zunächst durch Mangel an Konkurrenz zu erklären. Dieser Mangel an Konkurrenz ist aber im Unterschied zu den Bereichen nördlich der Alpen nicht primär edaphisch, sondern in sehr viel stärkerem Umfange klimatisch bedingt, da eben der submediterran-subkontinentale Klima charakter des Wallis nicht die artenreichen Laubmischwälder ermöglichte.

Trotz ihrer "ubiquistischen Bedürfnislosigkeit" (CHRIST 1919/20) kann eine "ökologische Strategie" der Föhre erkannt werden, die prinzipiell für alle Pinoiden gilt. Von allen heute vorkommenden Coniferen besitzt die Gattung Pinus schon auf Grund ihrer langen, dünnen und häufig bräunlich oder fahlgrünbräunlich gefärbten Nadeln ein sehr stark sklerophylles Gepräge. Obwohl dies ein genotypisch-konstitutionelles Merkmal ist und noch keine Anpassung an xerischere Umweltbedingungen bedeutet, so nehmen viele Pinus-Arten in auffälliger Weise klimatische und/oder edaphisch trockene Standorte ein. Andererseits sind Pinus-Arten häufig typische Vertreter oligotropher Moore, wobei freilich hier öko-physiologisch gesehen - nur ein scheinbarer Gegensatz zu den von vornherein als trocken anzusprechenden Standorten besteht, da eine gemeinsame Eigenschaft dieser extremen Standorte in ihrer schlechten Wasserkapazität gesehen werden muss. Dies kommt sehr deutlich bei der südostasiatischen Art Pinus merkusii zur Geltung (STEIN 1978). Es ist daher verständlich, dass diese charakteristische Eigenschaft vieler Pinus-Arten, sowohl trockene als auch nasse Standorte gut verwerten können, erst in den Grenzbereichen der Waldfähigkeit auch physiognomisch, d.h. landschaftsprägend, deutlich zum Ausdruck kommt (vgl. auch KLOETZLI 1975). Dies kann insbesondere in den asiatischen subkontinentalen Steppenrandbereichen, in den kontinentaleren Bereichen des sibirischen Tundragürtels sowie in den kontinental beeinflussten Teilen der Alpen deutlich beobachtet werden. .Dagegen dringen Pinus-Arten nur selten in die ozeanischeren Bereiche sommergrüner Laubwälder ein, und zwar bezeichnenderweise nur dort, wo die Bodenwasserverhältnisse zu einem permanenten physiologischen Stress in der Wasserversorgung führen können wie auf exponierten Felsgräten oder Mergelhängen, kiesigen oder sandigen Flussterrassen, grobem Bergsturzmaterial, oliotrophen Mooren, d. h. generell auf solchen Standorten, die für den jeweiligen grösseren Vegetationsbereich als extrem zu bezeichnen sind. Die anspruchslosen Pinus-Arten erweisen sich hier gegenüber den anderen vorhandenen Baumarten als konkurrenzüberlegen.

\section{Klimaökologische Gemeinsamkeiten der} Arealverbreitung der Weisstanne

Die Weisstanne ist in ihrem rezenten Verbreitungsmuster auf die montane Stufe konzentriert, wobei der arealmässige Schwerpunkt innerhalb der Schweiz im nördlichen Alpengebiet liegt (KUOCH 1954). Wenn man Verbreitungskarten der Vegetation und der wichtigeren waldbildenden Florenelemente (SCHMID 1961) mit Klimakarten (SCHUEPP in Atlas der Schweiz 1965) und Tabellen ausgewählter Klimawerte vergleicht, so können zweifellos die folgenden Ergebnisse gewonnen werden: 1) Das optimale Verbreitungsgebiet, in der die Weisstanne gesellschaftsbestimmend auftritt d. h. Gebiete und Standortsbereiche mit dem Abietetum typicum - sind durch reichliche Niederschläge (grösser $900 \mathrm{~mm}$ pro Jahr), einen relativ ausgeglichenen Gang der Jahres temperatur und durch eine erhöhte Nebelbereitschaft gekennzeichnet.

2) Der Jahresgang der Niederschläge in Gebieten mit reichlichen Weisstannenvorkommen lässt während der Vegetationsperiode oder zumindest längeren Teilen davon, insbesondere Mai-Juli, Monatswerte von mindestens $70 \mathrm{~mm}$, häufig von über $100 \mathrm{~mm}$ (= perhumide Jahres zeit nach WALTER/HARNICKEL/MUELLERDOMBOIS 1975) erkennen. Niederschlagssummen von weniger als $70 \mathrm{~mm}$ während eines Sommermonates scheinen eine kritische Grenze für das ökologische Optimum der Weisstanne zu sein. Freilich müssten hier ebenfalls Untersuchungen über die prozentuale Häufigkeit monatlicher Niederschlagssummen vorgenommen werden.

3) Abies alba dringt nur wenig in Gebiete mit kontinentalem Klimacharakter ein, sofern für diese die Parameter längerer Winterkälte und von Spätfrösten bevorzugt angewendet werden. Nur in Randbereichen von eindeutig ozeanisch beeinflussten Gebirgen mit sehr grossem 


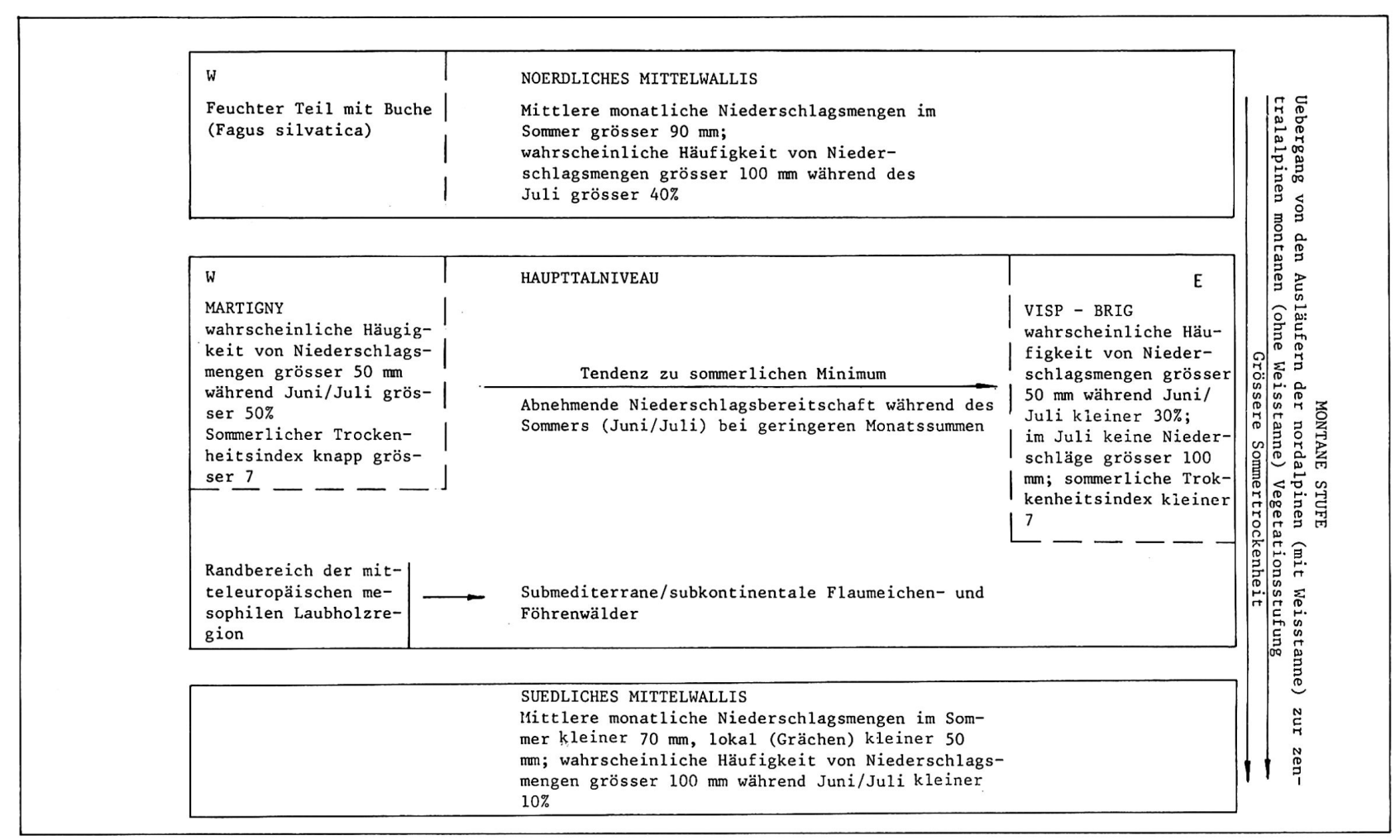

Tab. 5: Die räumlichen Beziehungen ökologisch signifikanter Niederschlagseigenschaften zu den Vorkommen von Flaumeichen, Föhren und Weisstannen im mittleren Wallis 
Weisstannenanteil können in Gebieten mit kontinentalem Klimacharakter Waldbestände mit bedeutendem Anteil von Abies alba vorkommen, so z. B. nach MUELLER/OBERDORFER/PHILIPPI (1974) der Labkraut/Tannenwald (Galio rotundifolii-Abietetum) und der artenreiche Tannenmischwald (Pyrolo-Abietetum) auf der Baar östlich des Schwarzwaldes. Räume mit extrem starker Spätfrostgefärdung (Münchner Schotterebene) müssen jedoch als

"Tannenausschlussgebiete" angesehen werden, in welchen A. alba innerhalb ihres grossräumigen Verbreitungs gebietes von Natur aus nicht vorkommt (WINDISCH-GRAETZ 1912; FIRBAS 1949; LANGER 1963).

4) In den wenigen Gebirgen des Mittelmeerraumes (Apenninen; Korsika; Balkanhalbinsel), in denen die Weisstanne vorkommt, nimmt sie eine regional meist umgrenzte Stellung ein und besetzt nur solche Standorte, die auch durch ihre weitere floristische Zusammensetzung eindeutig mitteleuropäisch-mesophile Konvergenzen aufweisen. Auf Korsika kommt A. alba zusammen mit der Rotbuche (Fagus silvatica) und der atlantischen Stechpalme (Ilex aquifolium) nur in dem gut beregneten westlichen Teil vor, während sie im zentralen Teil der Insel durch Kiefernwälder von Pinus pinaster und P. halepensis ersetzt wird (ELLENBERG 1964). Auch in Albanien kommt die Weisstanne nur in Rotbuchenwäldern der ausreichend Niederschlag erhaltenden Gebirge oberhalb von $1000 \mathrm{~m}$ bis $1500 \mathrm{~m}$ vor (MARKGRAF 1942). Das im mittleren Wallis abgeleitete Verbreitungsmuster der Weisstanne im ursächlichen Zusammenhang mit der Differenzierung in eine feuchte nördliche montane und eine sommertrokkenere südliche montane Stufe entspricht den beobachteten Gemeinsamkeiten der Weisstan nenverbreitung in Europa.

\section{Ergebnisse und Perspektiven für weitere Forschungen}

1) Es kann mit Hilfe von Flaumeiche, Föhre und Weisstanne, die unterschiedliche klimaökologische Ansprüche besitzen, eine räumlichklimaökologische Gliederung des mittleren Wallis innerhalb der Zentralalpen vorgenommen werden. Dabei sind verschiedene Gradienten, die sowohl durch charakteristische Eigenschaften der Niederschlagsverhältnisse als auch durch die Verteilung der drei Baumarten ( $\mathrm{Zu}$ - oder Abnahme der Vorkommenshäufigkeit) gekennzeichnet sind, dreidimensional übereinander gelagert (Tab. 5). Im Haupttalniveau ist von $W$ nach $E$ eine abnehmende Niederschlagsbereitschaft in den Sommermonaten zu erkennen, welche von einer Zunahme der Flaumeichen- und Föhrenvorkommen begleitet wird. Eine ähnliche Tendenz zur Sommertrockenheit vollzieht sich in der montanen Stufe in $\mathrm{N}-\mathrm{S}-\mathrm{Richtung}$, welche in vegetationsökologi- scher Beziehung von dem Ende der Weisstannenvorkommen begleitet wird.

2) Es ergibt sich, dass der Uebergang von der mitteleuropäischen zur zentralalpinen Vegetationsstufung im wesentlichen durch sommerliche Trockenheit bedingt wird, was hier bis zur oberen montanen Stufe bewiesen wurde. 3) Es ist mit Hilfe von Häufigkeitsanalysen monatlicher Niederschlagssummen - insbesondere während der pflanzenphysiologisch wichtigen sommerlichen Vegetationsperiode - sowie von Trockenperioden mit eindeutig definierten Schwellenwerten (in $\mathrm{mm}$ täglicher Niederschlag) möglich, das Verbreitungsmu ster von waldbildenden Baumarten schärfer zu fassen. Dies gilt sowohl für das Gesamtverbreitungsareal als auch für die räumliche Verteilung innerhalb einer ausreichend mit Stationen versehenen Raumeinheit. Es ist dies eine methodische Weiterführung, wenn auch keine prinzipielle Neuorientierung, der von KOEPPEN (1931) erarbeiteten Prinzipien zur effektiven Klassifikation der Klimate. 4) Beim überregionalen Vergleich von charakteristischen Vorkommensbereichen wichtiger bestansbildender Baumarten haben sich die von KOEPPEN benutzten Elemente der Mittelwertsklimatologie bewährt. Auch weitere neuere Arbeiten über die klimaökologischen Grundlagen mitteleuropäischer Waldgesellschaften (HARTMANN/SCHNELLE 1970; MITSCHERLICH 1971) bestätigen, dass zumindest in räumlich-vergleichendem Rahmen die mittleren Werte signifikanter Klimaeigenschaften unersetzlich sind. Das für die Bestandszusammensetzung so entscheidende Konkurrenzverhalten der Baumarten richtet sich - neben den hier nicht behandelten edaphischen Ursachen - weitgehend nach diesen über lange Zeiträume hinweg beobachteten Mittelwerten.

5) Die Grenze der bisherigen - und auch in dieser Arbeit angewendeten - Methoden besteht darin, dass die regionalen, vertikalen und expositionsbedingten Klimavariationen aus dem Beobachtungsmaterial von Klimastationen abgeleitet werden, die sämtlich ausserhalb der behandelten Bestandszusammensetzungen bzw. Waldtypen liegen. Die Bedeutung sehr unterschiedlicher Niederschlagsarten für die Wasserversorgung des Bestandes, der Interzep tionsvorgang und die Speicherkapazität sowie die Verteilung des Niederschlags im Bestand können zu unterschiedlichen Standortausprägungen führen. Diese Elemente werden mit den herkömmlichen Messmethoden der Stationen nicht erfasst und verlangen einen hohen und gewöhnlich zeitraubenden Geräteeinsatz. Es mag hier nicht entschieden werden, ob dieser spezielle Aufgabenbereich der Forstökologie zumindest teilweise in den Problemkreis der Vegetationsgeographie integriert werden soll. 6) Es hat sich bei der regionalen Analyse im 
mittleren Wallis herausgestellt, dass die Strahlungsverteilung im Gelände einen erheblichen Einfluss auf das Verbreitungsmuster der Flaumeiche besitzt. Es kann generell für Raumeinheiten' mit stark gegliedertem Relief, insbesondere auch in der subalpinen und alpinen Stufe von Hochgebirgen, abgeleitet werden, dass die geländeabhängige Strahlungsverteilung einen erheblichen Einfluss auf das Pflanzenleben und auf die räumliche Anordnung von wärmeliebenden Assoziationen (bei hohem Strahlungs genuss; z. B. Wa cholder-HeidekrautGesellschaft $=$ Callunetum mit Juniperus communis ssp. nana) oder feuchtigk eitsabhängigen Gesellschaften (bei geringem Strahlungsgenuss; z. B. Vaccinium-, Rhododendron- und Empetrum-Bestände mit reichem Moosunterwuchs) besitzt (DIRMHIRN 1964; TURNER 1966). Kartierungen von Hangbestrahlungen und entsprechenden Pflanzengesellschaften stellen sich demnach als ein wichtiges Ziel zukünftiger landschaftsökologischer Forschungen heraus.

1) In Spanien wird die Flaumeiche durch andere Arten (vor allem Qu.pyrenaica) ersetzt.

2) Zum sehr komplexen Problem der xeromorphen Struktur der transpirierenden Organe, insbesondere der Differenzierung von genotypischen Merkmalen und umweltbedingten Anpassungsformen vgl. WALTER 1968.

3) Allerdings spielt hier die wesentlich höhere Lage eine ebenfalls entscheidende Rolle für die höhere Niederschlagsbereitschaft; ferner zeigt auch der Vergleich mit dem westlichen Schwarzwald, dass oberhalb von 500-600m Eichen nicht mehr vorkommen.

\section{Literaturverzeichnis}

BACH, R. /KUOCH, R. /IBERG, R. 1954: Wälder der Schweizer Alpen im Verbreitungsgebiet der Weisstanne. Mitt. Schw. Anstalt f. d. forstl. Versuchsw. 30, 129-314

BA GNOULS, F. / GAUSSEN, H. 1952: L'indice xé rothermique. Bull. Ass. Géographes Français.

BAUMGARTNER, A. 1960: Gelände und Sonnenstrahlung als Standortfaktor am Gr. Falkenstein (Bayrischer Wald). Forstwiss. Centr. bl. 79, 286-297

BEZINGE, A. /BONVIN, G. 1974: Image du climat sur les Alpes. Bull. Murith. 91, 27-48

BIDER, M. / THAMS, J.C. 1951: Die Niederschlagsregime nord- und südwärts der Alpen. Arch. Met., Geophys. u. Bioklimat. 3, $123-148$
BOUET, M. 1948: L'insolation en Valais. Bull. Murith. 65, 82-94

BOUET, M. 1965: La pluie à Lausanne et à Sierre. Bull. Soc. Vaud. Sc. Nat. 69, 83-92

BRAUN-BLANQUET, J. 1961: Die inneralpine Trockenvegetation. Stuttgart

BRAUN-BLANQUET, J. /RICHARD, J. -L. 1949 : Groupements végétaux et sols du bassin de Sierre. Bull. Murith. Soc. Valais. Sci. Nat. 66, 106-138

BRAUN-BLANQUET, J. / ROUSSINE, N. / NEGRE, R. 1952: Les groupements vé gétaux de la France méditerranéenne. Montpellier

BRAUN-BLANQUET, J. / PALLMANN, H. / $\mathrm{BACH}, \mathrm{R}$. 1954: Pflanzensoziologis che und bodenkundliche Untersuchungen im Schweizerischen Nationalpark und seinen Nachbargebieten. Ergebnisse der wissenschaftlichen Untersuchungen des Schweiz. Nationalparks, Bd. IV, Liestal

BROCKMANN-JEROSCH, H. und M. 1910: Die natürlichen Wälder der Schweiz. Ber. Schweiz. Bot. Ges. 19, $171-225$

BURNAND, J. 1970: Vergleich von Waldgesellschaften im Gebiet der Bergsturzhügel von Siders. Dipl. -Arbeit Geobot. Inst. ETH, Zürich

BURNAND, J. 1976: Quercus pubescens Wälder und ihre ökologischen Grenzen im Wallis (Zentralalpen). Diss. rer. nat. Zürich

CATZEFLIS, J. / PRIMAULT, B. /STREHLER, H. 1972: Analyse de la pluviosité dans le Valais central, Veröff. Schweiz. Met. Zentr.anst. 28

CHRIST, H. 1919-20: Die Visp-Taler Föhrenregion im Wallis. Bull. Murith. 40, 187-273

DIRMHIRN, J. 1964: Das Strahlungsfeld im Lebensraum. Frankfurt a. M.

ELLENBERG, H. 1963: Vegetation Mitteleuropas mit den Alpen. Stuttgart

ELLENBERG, H. 1964: "Eigenbürtige" und "fremdbürtige" Vegetationsstufung auf Korsika. In: Beitr. z. Phytol. (Walter-Festschrift), Arb. Landw. Hochs ch. Hohenheim $30,145-154$

EMBERGER, L. 1955: Une classification biogéographique des climats. Rec. Trav. Labor. Bot. Montpellier, Sér. botan. , 7, 3-43

FIRBAS, F. 1949: Spät- und Nacheiszeitliche Waldgeschichte Mitteleuropas nördlich der Alpen. Bd. 1: Allgemeine Waldgeschichte, Jena

FLAHAULT, C. 1937: La distribution géographique des végétaux dans la région mé- 
diterranéenne française. Encycl. biol. 18, Paris

FLIRI, F. 1974: Die Alpen als Klimascheide: Vorstellungen und Wirklichkeit. In: Klimatologische Forschung (Festschrift für $\mathrm{H}$. FLOHN), Bonn. Met. Abh. 17, 417-426

FREITAG, H. 1964: Die Waldgesellschaften der Rouvière bei Montarnaud. Ein Beitrag zur Kenntnis der Vegetation des Languedoc. Arb. Landw. Hochsch. Hohenheim 30 (Walter-Fests chrift), 155-189

FREY,H. 1934: Die Walliser Felsensteppe. Diss. phil. Zürich

FURRER, E. 1942: Kleine Pflanzengeographie der Schweiz. Zürich

GAMS, H. 1927: Von den Follateres zur Dent de Morcles. Vegetationsmonographie aus dem Wallis. Beiträge zur geobotanischen Landesaufnahme der Schweiz 15, Bern

GEIGER, R. 1930: Mikroklima und Pflanzenklima. Berlin

GIACOBBE, A. 1949: Le basi concrete per una classificazione ecologica della vegetazione italiana. Forli

HAINARD, P. 1969: Signification é cologique et biogéographique de la répartition des essences forestieres sur l'adret valaisan. Boissiera 15, Genf

HAR TMANN, F. -K. /SCHNELLE, F. 1970: Klimagrundlagen natürlicher Waldstufen und ihrer Waldgesells chaften in deutschen Mittelgebirgen. Stuttgart

HESS, E. 1942: Die Waldungen des Kantons Wallis. Verh. Schweiz. Naturf. Ges. , 35-46

HEUER, I. 1949: Vergleichende Untersuchungen an den Föhrenbeständen des Pfynwaldes (Wallis). Versuch einer biocoenologischen Analyse. Beiträge zur geobotanischen Landesaufnahme der Schweiz 28, Bern

HOLTMEIER, F.-K. 1967: Die Verbreitung der Holzarten im Oberengadin unter dem Einfluss des Menschen und des Lokalklimas. Erdkunde 21, 249-258

HORVAT, I. 1954: Pflanzengeographische Gliederung Südosteuropas. Vegetatio 5-6, 434447

HORVAT, I. 1958: Laubwerfende Eichenzonen Südosteuropas in pflanzensoziologischer, klimatischer und bodenkundlicher Betrachtung. Angew. Pflanzensoz. 15, 50-62

HORVAT, I. 1962: Die Grenze der mediterranen und mitteleuropäischen Vegetation in Südosteuropa im Lichte neuer pflanzensoziologischer Forschungen. Ber. Dt. Bot. Ges. $75,91-104$
HUTTARY, J. 1950: Die Verteilung der Niederschläge auf die Jahreszeiten im Mittelmeergebiet. Met. Rundsch. 3, 111-119

IVERSEN, J. 1944: Vis cum, Hedera and Ilex as climatic indicators. Geol. Fören. Förh. 66, 463-483

JAKUCS, P. 1961 : Die phytozönologischen Verhältnisse der Flaumeichen-Buschwälder Südostmitteleuropas. Budapest

KELLER, P. 1935: Pollenanalytische Untersuchungen an Mooren des Wallis. Viert. jschr. Naturf. Ges. Zürich 80, 17-74

KLOETZLI, F. 1975: Oekologische Besonderheiten Pinus-reicher Waldgesellschaften. Schweiz. Ztschr. f. Forstwesen 126, 672-710

KOEPPEN, W. 1931: Grundriss der Klimakunde, Berlin/Leipzig

KUEMMEL, K. 1949: Die Stellung Südfrankreichs und der Krim im west- und ostmediterranen Vegetationsprofil. Bonn

KUHN, N. 1973: Frequenzen von Trockenperioden und ihre ökologische Bedeutung. Vierteljahresschr. Naturforsch. Ges. Zürich 118, 257-298

KUOCH, R. 1954: Wälder der Schweizer Alpen im Verbreitungsgebiet der Weisstanne. Mitt. Schweiz. Anst. Forstl. Versuchswesen $30,131-260$

LANG, G. 1955: Neue Untersuchungen über die spät- und nacheiszeitliche Vegetations geschichte des Schwarzwaldes. II. Das absolute Alter der Tannenzeit im Südschwarzwald. Beitr. z. naturk. Forsch. SW-Dt. land 14, 24-31

LANGER, H. 1963: Einwanderung und Ausbreitung der Weisstanne in Süddeutschland. Forstwiss. Centr. - bl. 82, 33-52

LARCHER, W. 1961: Jahresgang des Assimilations - und Respirationsvermögens von Olea europaea L. ssp. sativa HOFF, et LINK. , Quercus ilex L. und Quercus pubescens WILLD. aus dem nördlichen Gardaseegebiet. Planta 56, 575-606

LARCHER, W. /MAIR, B. 1968: Das Kälteresistenzverhalten von Quercus pubescens, Ostrya carpinifolia und Fraxinus ornus auf drei thermisch unterschiedlichen Standorten. Oecol. Plant. 3, 255-270

LARCHER, W. /MAIR, B. 1969: Die Temperaturresistenz als ökophysiologisches Konstitutionsmerkmal: 1. Quercus ilex und andere Eichenarten. Oecol. Plant. 4, 347-376

LAUER, W. 1952: Humide und aride Jahreszeiten in Afrika und Südamerika und ihre Beziehung zu den Vegetations gürteln. Bonn. Geogr. Abh. 9, 9-98 
LAUER, W. 1953: L'indice xérothermique ( $\mathrm{z}$. Frage d. Klimaindizes). Erdkunde 7, 48-52

LUEDI, W. 1955: Die Vegetationsentwicklung seit dem Rückzug der Gletscher in den mittleren Alpen und in ihrem nördlichen Vor land. Ber. Geobot. Insti. Rübel in Zürich f. d. Jahr 1954, 36-68

MARKGRAF, F. 1942: Die Südgrenze mittel'europäischer Vegetation auf der Balkanhalbinsel. Ber. Dt. Bot. Ges. 60, 118-127

MARKGRAF, F. 1952: Zur Abgrenzung der Mittelmeervegetation. Vegetatio 3, 324-325

MARKGRAF, F. 1969: Moorkundliche und vegetations geschichtliche Untersuchungen an einem Moorsee an der Waldgrenze im Wallis. Bot. Jb. $89,1-63$

MATTFELD, J. 1926: Die europäischen und mediterranen Abies-Arten. Die Pflanzenareale $1-2,22-29$

MATUSZKIEWICZ, W. 1962: Zur Systematik der natürlichen Kiefernwälder des mittel und osteuropäischen Flachlandes. Mitt. Flor. -soz. Arb. gem. N. F. 9, 145-186

MEUSEL, H. / JAEGER, E. /WEINERT, E. 1965: Vergleichende Chorologie der zentraleuropäischen Flora. Jena

MEYER, K. A. 1951 und 1955: Frühere Verbreitung der Holzarten und einstige Waldgrenze im Kanton Wallis. II. Mittelwallis. IV. Oberwallis. Mitt. Schweiz. Anst. f. d. forstl. Versuchswesen 27, 283-347 und 31 557-668

MITSCHERLICH, G. 1971: Wald, Wachstum und Umwelt. Eine Einführung in die ökologischen Grundlagen des Waldwachstums. Bd. 2: Waldklima u. Wasserhaushalt. Frankfurt a. M.

MUELLER, T. /OBERDORFER, E. / PHILIPPI, G. 1974: Die potentielle natürliche Vegetation von Baden-Württemberg. Beih. Veröf. Landesstelle f. Naturschutz und Landschaftspflege Baden-Württemberg 6, Ludwigsburg

OBERDORFER, E. 1948: Gliederung und Umgrenzung der Mittelmeervegetation auf der Balkanhalbinsel. Ber. Geobot. Inst. Rübel. f. d. J. 1947, 84-111

OBERDORFER, E. 1964: Der insubrische Vegetationskomplex, seine Struktur und $\mathrm{Ab}$ grenzung gegen die submediterrane Vegetation in Oberitalien und in der Südschweiz. Beitr. naturk. Forsch. Südwestdeuts chl. 23, 141-187

OZENDA, P. 1966: Perspectives nouvelles pour l'étude phytogéographique des Alpes du Sud. Docum. Carte Végét. Alp. IV, Grenoble
PALLMANN, H. 1934: Ueber Bodenbildung und Bodenserien in der Schweiz. Die Ernährung der Pflanze 30, 225-238

PALLMANN, H. /HAFFTER, P. 1933: Pflanzensoziologische und bodenkundliche Untersuchungen im Oberengadin. Ber. Schweiz. Bot. Ges. 42, 357-466

PRIMAULT, B. / ATZEFLIS, J. 1966: Du climat valaisan. La recherche agronomique en Suisse 5, 248-267

REGEL, C. 1947: Zur Frage der Grenze zwischen dem Mittelmeergebiet und Mitteleuropa in Griechenland. Ber. Geobot. Inst. Rübel. f. d. J. 1946, 15-22

REICHEL, E. 1949: Die Niederschlagshäufigkeit im Mittelmeergebiet. Met. Rundsch. 2, $129-142$

RIKLI, M. 1943: Das Pflanzenkleid der Mittelmeerländer. I. Bern

ROTEN, M. 1964: Recherches microclimatiques sur la vallée du Rhône en Valais. Diss. Univ. Fribourg. Sion

RUDOLPH, K. 1930: Grundzüge der nacheiszeitlichen Waldgeschichte Mitteleuropas Beih. Bot. Cbl. 47, 111-176

SCHMD, E. 1936: Die Reliktföhrenwälder der Alpen. Beiträge zur geobotanischen Landesaufnahme der Schweiz 21, Bern

SCHMID, E. 1961: Erläuterungen zur Vegetationskarte der Schweiz. Beitr. z. geobot. Landesaufnahme der Schweiz, H. 39, Bern

SCHNEIDER-CARIUS, K. 1955: Zur Frage der statistischen Behandlung von Niederschlagsbeoba chtungen. Zeits chr. f. Met. 9, 129-135, $193-202,266-271,299-300$

SCHNEIDER - CARIUS, K. / ESSENWANGER, O. 1955: Eigentümlichkeiten der Niederschlags verhältnisse im Norden und Süden der Schweizer Alpen, dargestellt durch die Niederschlagswahrscheinlichkeit von Basel, St. Gotthard und Lugano. Arch. f. Met. , Geophys. und Bioklim. B 7, 32-48

SCHUEPP, M. 1960 und 1968: Lufttemperatur. Klimatologie der Schweiz-C. Beih. Ann. Schweiz. Met. Zentr. -anstalt Jahrg. 1959, 1. Teil, H. 1 und Jahrg. 1967, 5. - 8. Teil, H. 8, Zürich

SCHUEPP, M. 1962: Sonnenscheindauer. Klimatologie der Schwejz I. Beih. Ann. Schweiz. Met. Zentr. -anst. 1961, Zürich

SCHWEINGRUBER, F. H. 1973: Föhrenwälder im Berner Oberland und am Vierwaldstättersee. Ber. Schweiz. Bot. Ges. 83, 175-204

STEIN, N. : Coniferen im westlichen malayischen Archipel. Studien zu ihrer Verbrei- 
tung und Oekologie innerhalb der vollhumiden südostasiatischen Tropen. Im Druck in der Reihe: BIOGEOGRAPHICA, Den Haag

TILL, O. 1956: Ueber die Frosthärte von Pflanzen sommergrüner Laubwälder. Flora $143,499-542$

TROLL, C. 1952: Das Pflanzenkleid der Tropen in seiner Abhängigkeit von Klima, Boden und Mensch. Tag. -ber. u. Wiss. Abh. Dt. Geogr. - T. Frankfurt a. M. 1951, 35-66

TROLL, C. 1957: Der Klima- und Vegetationsaufbau der Erde im Lichte neuer Forschungen. Jb. 1956 d. Akad. Wiss. u. Lit. Mainz, $216-229$

TROLL, C. 1961: Klima und Pflanzenkleid der Erde in dreidimensionaler Sicht. Die Naturwissenschaften $48,332-348$

TURNER, H. 1961: Jahresgang und biologische Wirkungen der Sonnen - und Himmelsstrahlung an der Waldgrenze der Oetztaler Alpen. Wetter und Leben 13, 93-113

TURNER, H. 1966: Die globale Hangbestrahlung als Standortfaktor bei Aufforstungen in der subalpinen Stufe. Mitt. Eidg. Anst. forstl. Versuchsw. 42, 109-168

UTTINGER, H. 1949: Die Niederschlagsmengen in der Schweiz 1901-1940. Zürich

UTTINGER, H. 1966 und 1970: Niederschlag. Klimatologie der Schweiz. - E. Beih. Ann. Schweiz. Met. Zentr. -anst. Jahrg. 19654. Teil, H. 6 und Jahrg. 1969, 5. -8. Teil, Zürich

\section{Literaturbesprechungen}

SULLIVAN Walter: Warum die Erde bebt, 383 S., 1977, Umschau-Verlag, Frankfurt/Ma in,DM 39.80, ISBN 3-524-00646-9.

Die Literatur zum Thema der Kontinentaldrift ist unübersehbar geworden. Trotzdem ist es verdienstvoll, dass Walter Sullivan's 'Continents in motion' ins Deutsche übersetzt worden sind. Der Wissenschaftsredaktor der NEW YORK TIMES verfügt über ein ausgesprochenes Darstellungstalent, und auch in der Uebersetzung liest sich die Geschichte der modernen globalen Tektonik von Wegener bis zu den neuesten internationalen Forschungsprojekten wie ein Roman. Provozierende Untertitel ("Die Erde als Ballon", "llagneti-
WALTER, H. 1968: Die Vegetation der Erde. Bd. II: Die gemässigten und arktischen Zonen. Stuttgart

WALTER, H. / LIETH, H. 1967: Klimadia gramm-Weltatlas. Jena

WALTER, H. /STRAKA, H. 1970: Arealkunde. Floristisch-historis che Geobotanik. Einführung in die Phytologie III/2, Stuttgart

WALTER, H. / HARNICKEL, E. / MUELLERDOMBOIS, D. 1975: Klimadiagramm-Karten der einzelnen Kontinente und die ökologische Klimagliederung der Erde. Stuttgart

WELTEN, M. 1958: Die spätglaziale und postglaziale Vegetationsentwicklung der Berner Alpen und Voralpen und des Walliser Haupttales. Veröffent. Geobot. Inst. Rübel. Zürich $34,150-158$

WENDELBERGER, G. 1963: Die Schwarzföhrenwälder Südosteuropas. Mitt. florist. soziol. Arb. -gem. 10, 130-148

WINDISCH-GRAETZ, H. von. 1912: Die ursprünglich natürliche Verbreitungsgrenze der Tanne in Süddeutschland. Naturwiss. $Z$. f. Forst- u. Landw. 10, 200-267

YOSHINO, M. M. 1964: Some local characteristics of the winds as revealed by windshaped trees in the Rhône valley in Switzerland. Erdkunde 18, 28-39

ZENTGRAF, E. 1949: Die Edeltanne. Allg. Forst- und Jagdztg. 121, 7-16

ZOLLER, H. 1964: Zur postglazialen Ausbreitungsgeschichte der Weisstanne in der Schweiz. Schweiz. Z. Forstwesen 115, $681-700$

sche Fussabdrücke" oder "Kann der Geist im Erdinnern gezähmt werden?") mögen den zukünftigen Wissenschafter zu leichtem Naserümpfen veranlassen. Die Darstellung ist indessen fundiert, und die Quellen genannt - ein für einen Journalisten bemerkenswertes Faktum!. Nicht minder faszinierend ist der Versuch, die Zukunft der Erde und der Menschheit anhand der heutigen Erkenntnisse zu skizzieren. Der dabei entwickelte Begriff von der "Sterblichkeit der Landschaften" bringt uns vielleicht dazu, in den grossen Weltproblemen sub specie aeternitatis, also weniger anthropozentrisch und gegenwartsbezogen zu denken.

GH 2/78

Walter Kyburz

Getreide-Elevatoren mit Weizenmahden bei Riceton, Saskatchewan

Foto aus dem Bildband: Walter Weiss, KANADA - von Neufundland zum Pazifik. 208 Seiten, 97 mehrfarbige, 39 einfarbige Tafeln, 12 Kartenskizzen, Format $24 \times 30 \mathrm{~cm}$, Fr. 78.-. (c) Kümmerly+Frey, Geographischer Verlag, Bern 